\title{
Indium phosphide magic-sized clusters: chemistry and applications
}

\author{
Yongju Kwon ${ }^{1}$ and Sungjee Kim (1)
}

\begin{abstract}
This paper reviews developments in the synthesis of InP quantum dots (QDs) and research on InP magic-sized clusters (MSCs), which occur as intermediates during the growth of InP QDs. We classify research on InP MSCs into (1) synthesis and characterization and (2) applications. Several InP MSCs have been developed using low reaction temperatures or strong binding ligands. InP MSCs passivated by carboxylate, amine or phosphonate ligands and InP MSCs that incorporate heterogeneous atoms ( $\mathrm{Cl}$ or $\mathrm{Zn}$ ) have been synthesized. We also review tools to characterize MSCs and show the intermediate characteristics of InP MSCs. InP MSCs have been used as synthesis precursors for spherical InP QDs and nonspherical InP nanostructures, such as elongated, branched, and hyperbranched/dendrimer-like morphologies. We also present MSC-mediated growth mechanisms involving fragments, monomers or other nanoclusters. Research on the chemistry and applications of InP MSCs has uncovered the formation process of InP nanomaterials. Developments in the luminescent properties of InP MSCs, InP QDs and elongated InP nanostructures would be useful in displays.
\end{abstract}

\section{Introduction}

Colloidal semiconductor quantum dots (QDs) have unique optical, physical, and chemical properties. Rapid development in synthesis methods has led to the production of high-quality QDs, which have been used in efficient photovoltaic devices, light-emitting diodes, photodetectors, and biomedical imaging systems ${ }^{1-4}$.

Indium phosphide (InP) QDs are direct-bandgap III-V compound semiconductors (bulk bandgap: $1.35 \mathrm{eV}$ ) that can be made to emit from the blue $(\sim 465 \mathrm{~nm})$ to the nearinfrared $(\sim 750 \mathrm{~nm})$ region by simply tuning their size $\mathrm{s}^{5-7}$. InP QDs have relatively low toxicity, so they have the potential to be commercially utilized, especially in displays $^{4,8-10}$.

InP magic-sized clusters (MSCs) were recently discovered as intermediates during the growth of $\operatorname{InP} \mathrm{QDs}^{10-12}$. InP MSCs possessing high thermal stability may act as barriers to the growth of InP QDs ${ }^{13}$. Research on understanding the growth pathway, including InP MSCs is being actively

\footnotetext{
Correspondence: Sungjee Kim (sungjee@postech.ac.kr)

'Department of Chemistry, Pohang University of Science and Technology,

Pohang 37673, South Korea
}

conducted for the synthesis of high-quality InP QDs. We believe that unmet challenges in InP QD synthesis, such as monodisperse QDs and precise morphology control, can be overcome by exploring the nature of InP MSCs. Isolation and characterization of InP MSCs and studies on their reaction pathways would be key to controlling the synthetic pathways. In this review, developments in the synthesis of InP QDs and research on InP MSCs are summarized. We classify research on InP MSCs into (1) synthesis and characterization and (2) applications.

\section{History of synthesis of InP QDs and InP magic-sized clusters}

Methods to synthesize InP QDs are not well developed. Moreover, the broad emission profile of InP QDs is a demerit. The band-edge PL of a single InP QD has a full width at half maximum (FWHM) of $14 \mathrm{~nm}$ at $613 \mathrm{~nm}$, so narrow emission of ensemble InP QDs is not limited on a fundamental level ${ }^{14}$.

An inhomogeneous size distribution may be the main reason for the broad emission peak, so methods to synthesize monodisperse InP QDs have been sought ${ }^{5,15,16}$. 
To narrow the size distribution of InP QDs, some studies have attempted to control the reactivity of molecular precursors. The commonly used tris(trimethylsilyl)phosphine $\left((\mathrm{TMS})_{3} \mathrm{P}\right)$ is too reactive and leads to inseparable nucleation and growth steps, so it has been replaced by less-reactive precursors such as tris(trimethylgermyl) phosphine ${ }^{15,16}$, phosphine $\left(\mathrm{PH}_{3}\right)^{17}$, aminophosphine ${ }^{18,19}$, and others ${ }^{20-23}$. However, the use of these different molecular precursors did not lead to any significant decrease in the size variation of the synthesized InP QDs. To date, the emission properties of InP QDs have not been reduced to a level comparable to that of Cd-based QDs. To the best of our knowledge, the narrowest ensemble emission peak of InP QDs reported thus far is an $\sim 35 \mathrm{~nm}$ FWHM, whereas that of ensemble CdSe can be $\sim 20 \mathrm{~nm}^{4,24-26}$. This wide FWHM of InP QDs motivates research to understand the mechanism of InP QD growth.

Nucleation and growth stages may be key steps that determine the uniformity of InP QDs. The classical LaMer model of nucleation and growth kinetics does not cover the whole of nanomaterial growth ${ }^{27}$, and nonclassical growth mechanisms have been recently reported ${ }^{28-30}$. InP QDs grow via the formation of intermediate states, which have been called MSCs, molecular nanoclusters or atomically precise nanoclusters ${ }^{10}$. Here, nanoclusters can be defined as small-sized nanocrystals in the range of $2 \mathrm{~nm}$ or less ${ }^{31,32}$. An MSC is composed of a specific number of atoms that are arranged to form highly stable structures with a size $<2 \mathrm{~nm}$ (Fig. 1) ${ }^{31-34}$. MSCs are generally identified by the wavelength of the persistent lowest energy electronic transition (LEET). The optical properties of MSCs are clearly different from those of general QDs in that the narrow bandwidth of the LEET is a result of single-sized products.

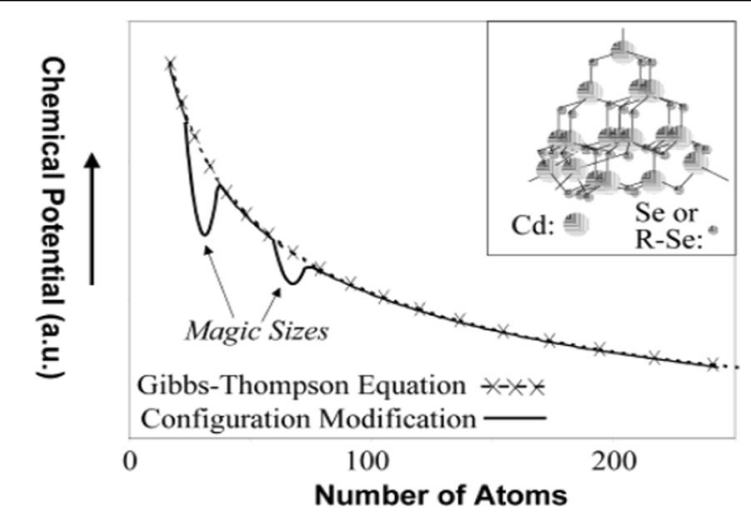

Fig. 1 Schematic illustration of the relative chemical potential in the small size regime of NPs. The relative chemical potential of crystals/clusters in the extremely small size regime has dependence of the size and configuration. Adapted with permission from ref. ${ }^{34}$. Copyright 2002 American Chemical Society.
MSCs are quite difficult to control. The formation and dissolution of MSCs also limit the rate of formation of QDs (Fig. 1) $)^{34,35}$. InP MSCs have been captured as intermediates during the growth of InP QDs, and the mass of the InP MSCs has been detected up to $30{ }^{\circ} \mathrm{C}^{13}$; the results indicated that the high thermal stability of InP MSCs influences the growth of InP QDs. This insight led to the isolation and characterization of various InP MSCs for study to increase the understanding of the growth mechanisms of InP QDs.

\section{Synthesis and characterization of chalcogenide MSCs and InP MSCs}

Three general synthesis pathways for MSCs have been developed: (1) the interruption method ${ }^{36-38}$, (2) the lowtemperature method $^{39-41}$, and (3) the size-focusing method $^{42-44}$. The interruption method obtains MSCs by interrupting the growth of large QDs by injecting a cold reagent immediately after hot injection. The lowtemperature method grows MSCs very slowly, so thermodynamically favorable MSCs form. The size-focusing method includes three steps: growth of large QDs at high temperature, quenching of the reaction to stop the growth of large QDs, and isothermal annealing to shrink the large QDs via size focusing for growth of MSCs.

Several InP MSCs with different surfactants have been synthesized using the low-temperature method. InP MSCs capped with a carboxylate ligand that had a dominant absorption peak at wavelength $\lambda=386 \mathrm{~nm}$ (386-InP MSCs) were discovered using indium acetate (In $\left.(\mathrm{Ac})_{3}\right)$, myristic acid (HMy), and (TMS $)_{3} \mathrm{P}$ in 1-octadecene (ODE) at $100^{\circ} \mathrm{C}^{10,35}$. The 386 -InP MSC was identified as $\mathrm{In}_{37} \mathrm{P}_{20}\left(\mathrm{O}_{2} \mathrm{CR}\right)_{51}$ (R: alkyl group) by single-crystal X-ray diffraction (SCXRD); because of its definite MSC structure, it has become the most-studied InP MSC system ${ }^{45}$. MSCs with amine ligands have been synthesized at $70{ }^{\circ} \mathrm{C}^{12}$. Phosphonate-capped InP MSCs have been grown at $370{ }^{\circ} \mathrm{C}^{10}$. Strong binding ligands such as phosphonic acid could be useful for the growth of InP MSCs even at high temperature.

InP MSCs that incorporate heterogeneous atoms have been reported ${ }^{11,46,47}$. InP MSCs incorporating chlorine (InP:Cl MSCs) or zinc (InP:Zn MSCs) are considered in this review as representatives.

To understand the optical, structural, and compositional properties of InP MSCs, characterization studies are necessary. Several methods to characterize semiconductor MSCs have been developed (Table 1).

Confirmation of characteristic features in absorption spectra is the most common method used to identify MSCs after synthesis. The absorption features of MSCs are very unique because they have a very narrow width at the excitonic transition. In addition, their absorption peaks persistently appear at fixed wavelengths. The optical 
Table 1 Characterization methods of semiconductor magic-sized clusters.

\begin{tabular}{|c|c|c|c|c|c|c|c|c|c|}
\hline \multicolumn{2}{|l|}{ Optics } & \multicolumn{2}{|l|}{ Structure } & \multicolumn{2}{|l|}{ Mass } & \multicolumn{2}{|c|}{ Size/Morphology } & \multicolumn{2}{|c|}{ Composition } \\
\hline Methods & Examples & Methods & Examples & Methods & Examples & Methods & Examples & Methods & Examples \\
\hline Steady-state absorption & $\begin{array}{l}\operatorname{InP}^{11,45} \\
\operatorname{InAs}^{12} \\
\mathrm{CdS}^{123} \\
\mathrm{CdSe}^{124} \\
\mathrm{CdTe}^{125} \\
\mathrm{Cd}_{3} \mathrm{P}_{2}^{126} \\
\mathrm{Cd}_{3} \mathrm{As}_{2}{ }^{127} \\
\mathrm{ZnSe}^{36} \\
\mathrm{ZnTe}^{128} \\
\mathrm{PbSe}^{33} \\
\mathrm{Ag}_{2} \mathrm{~S}^{55}\end{array}$ & SCXRD & $\begin{array}{l}\ln \mathrm{P}^{45} \\
\mathrm{CdSe}^{39,129}\end{array}$ & MALDI-TOF & $\begin{array}{l}\ln P^{13} \\
\mathrm{CdS}^{28} \\
\mathrm{CdSe}^{130} \\
\mathrm{CdZnSe}^{93,131}\end{array}$ & TEM & $\begin{array}{l}\ln P^{11,40} \\
\mathrm{CdS}^{132} \\
\mathrm{CdSe}^{133} \\
\mathrm{Cd}_{3} \mathrm{P}_{2}^{126}\end{array}$ & $\mathrm{ICP}$ & $\begin{array}{l}\ln P^{11,134} \\
\operatorname{CdS}^{59}\end{array}$ \\
\hline Steady-state PL & & pXRD & $\begin{array}{l}\ln P^{11,12,71} \\
\mathrm{CdS}^{11,50} \\
\mathrm{CdSe}^{59,135} \\
\mathrm{ZnTe}^{77}\end{array}$ & & & STEM & $\mathrm{CdSe}^{57}$ & EDS & $\begin{array}{l}\ln \mathrm{P}^{11,58} \\
\mathrm{CdSe}^{94} \\
\mathrm{Cd}_{3} \mathrm{P}_{2}^{126}\end{array}$ \\
\hline & & Raman & $\begin{array}{l}\operatorname{InP}{ }^{11,71} \\
\mathrm{CdS}^{50} \\
\mathrm{CdSe}^{66}\end{array}$ & ESI-MS & $\begin{array}{l}\mathrm{CdS}^{53} \\
\mathrm{CdSe}^{136} \\
\mathrm{CdTe}^{30}\end{array}$ & SAXS & $\begin{array}{l}\mathrm{CdSe}^{137} \\
\mathrm{CdTe}^{30} \\
\text { CdTeSe }^{40}\end{array}$ & RBS & $\mathrm{CdS}^{60}$ \\
\hline Transient absorption & $\begin{array}{l}\mathrm{InP}^{11} \\
\mathrm{CdSe}^{92} \\
\mathrm{ZnTe}^{77}\end{array}$ & PDF & $\begin{array}{l}\ln P^{10} \\
\mathrm{CdS}^{50} \\
\mathrm{CdSe}^{39}\end{array}$ & & & & & TGA & $\mathrm{CdS}^{59}$ \\
\hline Transient PL & $\begin{array}{l}\operatorname{lnP}^{11} \\
\mathrm{CdSe}^{81} \\
\mathrm{ZnTe}^{98}\end{array}$ & EXAFS & $\begin{array}{l}\mathrm{CdS}^{67} \\
\mathrm{CdSe}^{137}\end{array}$ & LDI-MS & $C d S e^{54,138}$ & Surface ligand & & DFT & \\
\hline & & XANES & $\mathrm{CdSe}^{49}$ & & & Methods & Examples & Examples & \\
\hline PLE & $\begin{array}{l}\ln P^{11} \\
\mathrm{CdSe}^{81,135} \\
\mathrm{CdTe}^{139} \\
\text { ZnTe }^{39}\end{array}$ & XPS & $\begin{array}{l}\ln P^{11} \\
\operatorname{CdS}^{50} \\
C d S e^{140}\end{array}$ & & & NMR & $\begin{array}{l}\ln P^{11,58,76} \\
\mathrm{CdSe}^{51}\end{array}$ & $\begin{array}{l}\ln P^{62} \\
\mathrm{CdSS}^{61,141} \\
\mathrm{ZnS}^{142} \\
\mathrm{PbS}^{143}\end{array}$ & \\
\hline MCD & $\begin{array}{l}\mathrm{CdSe}^{48,49} \\
\mathrm{CdZnSe} \mathrm{S}^{93}\end{array}$ & $\begin{array}{l}\text { Solid-state } \\
\text { NMR }\end{array}$ & $\mathrm{CdSe}^{51}$ & GPC & $\ln P^{11}$ & FT-IR & $\begin{array}{l}\ln P^{58} \\
\mathrm{CdSe}^{144}\end{array}$ & & \\
\hline
\end{tabular}

PLE photoluminescence excitation, MCD magnetic circular dichroism, SCXRD, single-crystal X-ray diffraction, $p X R D$ powder X-ray diffraction, $P D F$ pair distribution function, EXAFS extended X-ray absorption fine structure, XANES X-ray absorption near-edge structure, XPS X-ray photoelectron spectroscopy, NMR nuclear magnetic resonance, MALDI-TOF, matrix-assisted laser desorption/ionization time-of-flight, ESI-MS, electrospray ionization mass spectroscopy, LDI-MS laser desorption/ionization mass spectroscopy, GPC gel-permeation chromatography, TEM transmission electron microscopy, STEM scanning TEM, SAXS small-angle X-ray scattering, FT-IR Fouriertransform infrared spectroscopy, ICP inductively coupled plasma, EDS energy-dispersive spectroscopy, RBS Rutherford backscattering spectroscopy, TGA thermogravimetric analysis, DFT density functional theory.

properties of MSCs can be investigated using steady-state absorption/PL, transient absorption (TA)/PL, and photoluminescence excitation (PLE) spectroscopy ${ }^{11}$. Especially for the magneto-optical properties of doped MSCs, magnetic circular dichroism (MCD) can be useful ${ }^{48,49}$.

The structure can be analyzed using SCXRD ${ }^{45}$. SCXRD analysis upon successful crystallization of MSC samples can reveal the whole structure of MSCs, but the preparation of single crystals fit for SCXRD is difficult for several reasons, including the existence of MSC isomers and the intrinsic low symmetry of MSCs. Powder X-ray diffraction (pXRD), Raman spectroscopy, the pair distribution function (PDF) technique, X-ray absorption spectroscopy (XAS), including extended X-ray absorption fine structure (EXAFS) and X-ray absorption near-edge structure (XANES), X-ray photoelectron spectroscopy (XPS), and solid-state nuclear magnetic resonance (NMR) spectroscopy have been used as tools to determine MSC structures, where each of the analytical tools may provide complementary pieces of information for MSC structures ${ }^{11,49-51}$. 
The mass of MSCs can be determined using matrixassisted laser desorption/ionization time-of-flight (MALDITOF) mass spectrometry, electrospray ionization mass spectrometry (ESI-MS), laser desorption/ionization mass spectrometry (LDI-MS), and ion-mobility mass spectrometry (IM-MS) ${ }^{13,52-54}$. Despite the soft ionization method, MSCs can often be fragmented, and broad mass peaks can appear $^{30,46}$. Samples of MSCs often exist as mixtures and require isolation of specific kinds using, for example, gelpermeation chromatography (GPC), high-performance liquid chromatography (HPLC), and thin layer chromatography (TLC) ${ }^{11,55,56}$.

The size and morphology of MSCs can also be analyzed using transmission electron microscopy (TEM) or scanning transmission electron microscopy (STEM) $)^{11,57}$. However, the limited resolution for small-sized MSCs and sample degradation issues under electron beam irradiation remain challenges. Synchrotron small-angle X-ray scattering (SAXS) has also been used to attempt to measure MSC sizes $^{58}$.

The compositions of MSCs can be analyzed using inductively coupled plasma (ICP) spectroscopy, energydispersive spectroscopy (EDS), Rutherford backscattering spectroscopy (RBS), and thermogravimetric analysis (TGA) ${ }^{11,59,60}$. The inorganic components of MSCs are mainly determined using these tools. Surface ligand properties such as quantity and binding mode can be determined using ${ }^{1} \mathrm{H}$ NMR, ${ }^{13} \mathrm{C}$ NMR, or Fouriertransform infrared spectroscopy (FT-IR) $)^{11,58}$.

Density functional theory (DFT) calculations can also help elucidate $\mathrm{MSCs}^{61}$. The atomically resolved structure of MSCs revealed by SCXRD can be used for DFT calculations. For example, the correlation of electronic or geometric structural properties with energetics can be identified, which helps interpret the experimental results ${ }^{62}$. Unknown MSC structures can also be expected via modeling using such calculations ${ }^{61,63-65}$. Much experimental data, such as UV-vis absorption, PDF, EXAFS, and Raman spectra, can be compared to models calculated from simulated data ${ }^{50,63,66-68}$.

\section{Use of chalcogenide MSCs and InP MSCs}

Isolation and characterization of MSCs can yield an understanding of their growth process and suggest methods to control the intermediates ${ }^{11}$. Chalcogenide MSCs have been used as single-source precursors (SSPs) ${ }^{69}$. Precisely controlled growth was achieved for $2.5-9.0 \mathrm{~nm}$ CdSe and 3.0-5.0 $\mathrm{nm} \mathrm{ZnSe}$ QDs by using $\left(\mathrm{Li}_{4}\right)_{4}\left[\mathrm{M}_{10} \mathrm{Se}_{4}(\mathrm{SPh})_{16}\right]$ $(\mathrm{M}=\mathrm{Cd}, \mathrm{Zn} ; \mathrm{SPh}=$ thiophenolate $)$ as SSPs. This synthesis approach has advantages of size control, narrow size distribution, and large-scale production of QDs. Furthermore, high-quality QDs grew even at a low reaction temperature $T_{\mathrm{R}}<200^{\circ} \mathrm{C}$ because MSCs and their fragments could function as seeds for QDs without a nucleation process ${ }^{70}$.
III-V semiconductor QDs such as InP and InAs have been synthesized using nanoclusters as $\operatorname{SSPs}^{10,25,71-76}$. Sizefocused InAs QDs have been synthesized using InAs MSC-mediated growth. Rapid dissolution of MSCs may be the key to avoiding a broad size distribution that occurs via Ostwald ripening ${ }^{74}$. Highly monodispersed InAs QDs $(<10 \%$ size deviation) can be synthesized using $\sim 1.8 \mathrm{~nm}$ InAs nanoclusters as precursors ${ }^{73}$, which may be more stable than molecular arsenic precursors and are therefore suitable as growth precursors. Variously sized InAs QDs can be synthesized using intermediate nanoclusters as growth precursors to maintain the initial homogeneity ${ }^{75}$.

Semiconductor MSCs are often intermediates during the growth of nanorods, nanowires, nanoribbons, or nanoplatelets ${ }^{34,38,77-79}$. MSCs have been considered to be useful building blocks for the construction of semiconductor nanostructures (NSs) of diverse morphology (Fig. 2a) ${ }^{34}$. Semiconductor MSCs or their fragments can grow via oriented attachment in certain directions (Fig. 2b, c) ${ }^{78,79}$. Branched InP NSs (BNSs) and hyperbranched InP NSs (HBNSs)/dendrimer-like NSs (DLNSs) have been synthesized using InP MSCs as precursors ${ }^{80}$.

The luminescence of chalcogenide MSCs and InP MSCs has strong band-edge emission or broad white light emission that involves band edges and traps (Fig. 2d, e) $)^{12,54,81}$. The emission properties of MSCs have been exploited in luminous applications such as LEDs $^{82}$. Blue-emitting CdSe MSCs with emission peaks at $\lambda=420 \mathrm{~nm}$ or $450 \mathrm{~nm}$ have been reported; their quantum yield (QY) was improved from 5 to $24 \%$ by simple surface treatment (Fig. 2d) ${ }^{54,83}$.

Band-edge emission of MSCs is usually associated with broad trap emission at $400 \leq \lambda \leq 800 \mathrm{~nm}$. White emission with $\mathrm{QY}=27 \%$ was achieved by additional ligand treatment; the MSCs were used as white-light phosphors (Fig. 2e, f) ${ }^{57,81,82,84-87}$. PbSe MSCs with $50 \leq \mathrm{QY} \leq 90 \%$ were achieved with no posttreatment ${ }^{88}$, and ultrasmall CdSe nanoclusters with $\mathrm{QY}=6.3 \%$ (in water) were used for intracellular imaging (Fig. $2 \mathrm{~g})^{89}$.

\section{Synthesis and characterization of InP MSCs InP MSCs with different surface ligands}

Ultrasmall InP nanoclusters of size $1.5-2.3 \mathrm{~nm}$ were successfully synthesized in $2001^{90}$. A chloroindium oxalate complex and (TMS) ${ }_{3} \mathrm{P}$ were used as synthesis precursors; trioctylphosphine oxide (TOPO) and trioctylphosphine (TOP) were added as colloidal stabilizers/solvents. To synthesize ultrasmall $\mathrm{InP}$ nanoclusters, $T_{\mathrm{R}}$ was dropped to $100-220^{\circ} \mathrm{C}$ instead of $300-400^{\circ} \mathrm{C}$, which is suitable for the growth of InP QDs. In addition, volatile stabilizers such as pyridine, tributylphosphine, hexylamine, or trioctylamine were used instead of the heavy coordinating solvents TOPO and TOP. The low $T_{\mathrm{R}}$ inhibits the growth of nanoclusters into large QDs and is, therefore, one of the 
(a)

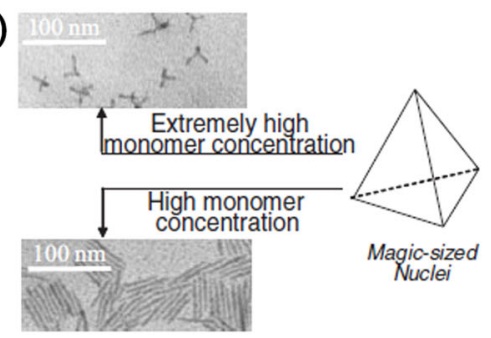

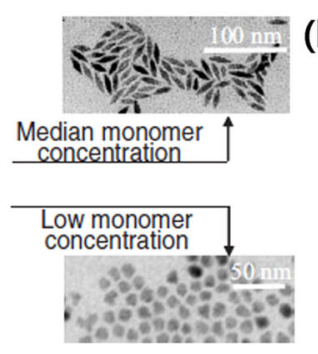

(b)
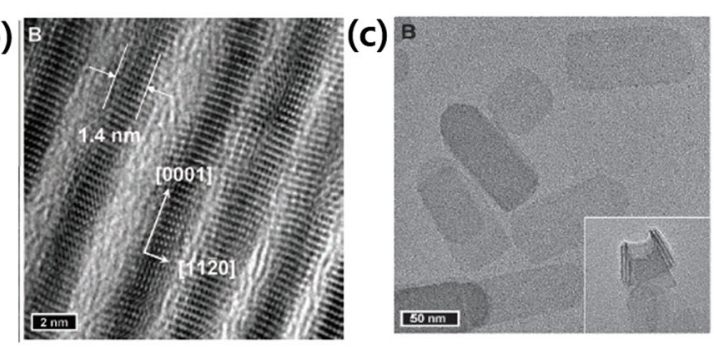

(d)
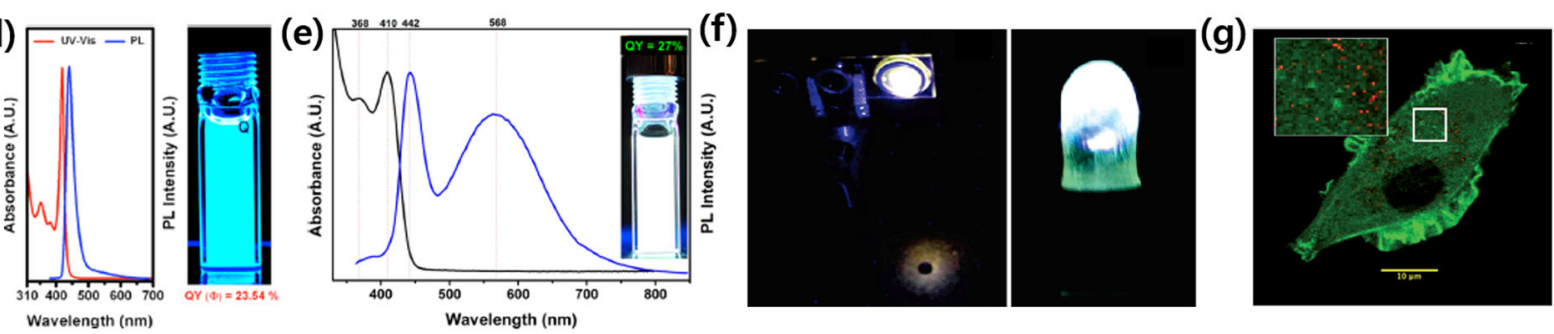

Fig. 2 Application of semiconductor MSCs. a Monomer-concentration-dependent, MSCs-mediated growth of CdSe nanocrystals. Adapted with permission from ref. ${ }^{34}$. Copyright 2003 WILEY VCH. b TEM image of CdSe nanoribbons synthesized from CdSe MSCs. Adapted with permission from ref. ${ }^{79}$. Copyright 2009 WILEY VCH. c TEM image of CdSe single-layered nanosheets synthesized from CdSe MSCs. Adapted with permission from ref. $^{78}$. Copyright 2006 American Chemical Society. d UV-vis absorption and PL spectra of oleic acid/trioctylphosphine-capped CdSe MSCs. Adapted with permission from ref. ${ }^{54}$. Copyright 2014 American Chemical Society. e UV-vis absorption and PL spectra of oleylamine capped CdSe MSCs. Adapted with permission from ref. ${ }^{81}$. Copyright 2015 American Chemical Society. $\mathbf{f}$ Thin film of white-light CdSe MSCs in polyurethane excited by a frequency-doubled titanium: sapphire laser $(400 \mathrm{~nm})$ with white light clearly seen reflecting off the table surface (left) and a 5-mm commercial UV LED $\left(400 \mathrm{~nm}\right.$ ) illuminating a thin coating of white-light CdSe MSCs in polyurethane (right). Adapted with permission from ref. ${ }^{85}$. Copyright 2005 American Chemical Society. $\mathbf{g}$ Representative differential interference contrast image of a green fluorescence protein transfected fibroblast cell incubated with $100 \mathrm{nM} \mathrm{TPP}$-treated PEG $_{18}$-thiolate-protected CdSe nanoclusters (red) and green fluorescence protein transfected cell. Adapted with permission from ref. ${ }^{89}$. Copyright 2014 The Royal Society of Chemistry.

most important conditions to yield small nanoclusters, including MSCs ${ }^{30,39,85}$. Optical characterization of synthesized ultrasmall InP nanoclusters showed an excitonic absorption peak at $\lambda \sim 400 \mathrm{~nm}$ that is quite narrow compared to the general absorption features of InP QDs.

As common precursors for the synthesis of InP QDs, an indium carboxylate complex and (TMS) ${ }_{3} \mathrm{P}$ have been developed since $2009^{6}$. 386 -InP MSCs were observed at $138 \leq T_{\mathrm{R}} \leq 208^{\circ} \mathrm{C}$ during the growth of InP QDs (Fig. 3a). Subsequent SCXRD analysis demonstrated that 386-InP MSCs have the formula $\operatorname{In}_{37} \mathrm{P}_{20}\left(\mathrm{O}_{2} \mathrm{CR}\right)_{51}$ (R: alkyl group) (Fig. 3b $)^{45}$. 386-InP MSCs have a large FWHM of $\sim 40 \mathrm{~nm}$ at $\lambda=386 \mathrm{~nm}$ due to vibronic coupling. The absorption peak at $386 \mathrm{~nm}$ narrows significantly when the $386-\mathrm{InP}$ MSC solution is cooled to $100 \mathrm{~K}$ (Fig. 3c).

To produce 386-InP MSCs, various synthesis conditions, including $T_{\mathrm{R}}$, the precursor concentration, and the nature of ligands, have been investigated ${ }^{35}$. Myristatecapped 386-InP MSCs are generally synthesized at $100^{\circ} \mathrm{C}$ and are stable up to $208^{\circ} \mathrm{C}$. Survival at this high temperature indicates that 386-InP MSCs are heavily involved in the growth of InP QDs.

Synthesis of 386-InP MSCs requires a concentration of the indium carboxylate complex that is least two times that of $(\mathrm{TMS})_{3} \mathrm{P}$ due to the cation-rich composition of MSCs.
Alkyl chain lengths of carboxylate ligands from butanoic acid $\left(C_{4}\right)$ to stearic acid $\left(C_{18}\right)$ have been used in the synthesis of 386-InP MSCs. When the alkyl chain is short, 386-InP MSCs form quickly but survive only at low temperature; for example, 386-InP MSCs with hexanoic acid $\left(\mathrm{C}_{6}\right)$ can survive up to $158^{\circ} \mathrm{C}$, whereas $386-\mathrm{InP}$ MSCs with HMy $\left(\mathrm{C}_{12}\right)$ can survive up to $208^{\circ} \mathrm{C}$. Prior to the formation of 386-InP MSCs, molecular precursors and monomers with shortlength ligands may be unstable in organic solvents, which leads to fast nucleation. After the formation of 386-InP MSCs, long alkyl chain ligands on the surface increase the solubility of the MSCs and thereby help stabilize the structure even at high temperature.

Clear identification of the structure of 386-InP MSCs allowed characterization of the details of the inorganic core and surface ligand binding mode ${ }^{45,71}$. The $\left[\operatorname{In}_{21} \mathrm{P}_{20}\right]^{3+}$ core is located in the center of $\operatorname{In}_{37} \mathrm{P}_{20}\left(\mathrm{O}_{2} \mathrm{CR}\right)_{51}$ with pseudo- $\mathrm{C}_{2 \mathrm{v}}$ symmetry (Fig. $3 \mathrm{~d}$ ). The average In-P bond length in the $\left[\operatorname{In}_{21} \mathrm{P}_{20}\right]^{3+}$ core is $2.528 \AA$, whereas that in the zinc-blende lattice is $2.541 \AA$. Interestingly, the structure of $386-\operatorname{InP}$ MSCs is not zinc-blende or wurtzite. 386-InP MSCs contain numerous interlocking polytwistane units ${ }^{71}$. A twistane of $\mathrm{In}_{5} \mathrm{P}_{5}$ has a twisted-boat conformation (Fig. 3e). An $\operatorname{In}_{13} \mathrm{P}_{14}$ polytwistane subunit with $\mathrm{T}$ symmetry also occurs in 386InP MSCs (Fig. 3f). The surface of 386-InP MSCs bears an 


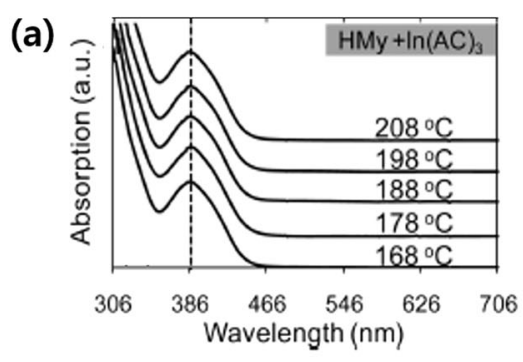

(b)

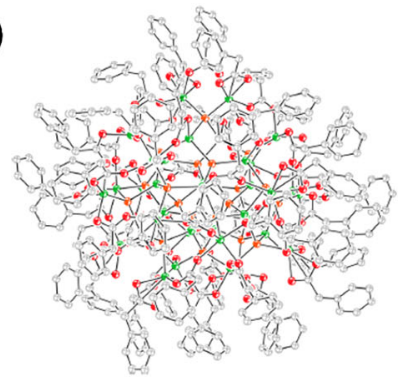

(d)

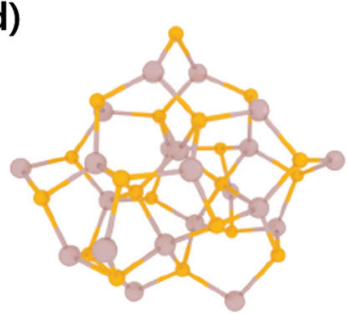

(e)

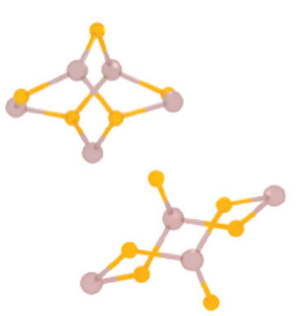

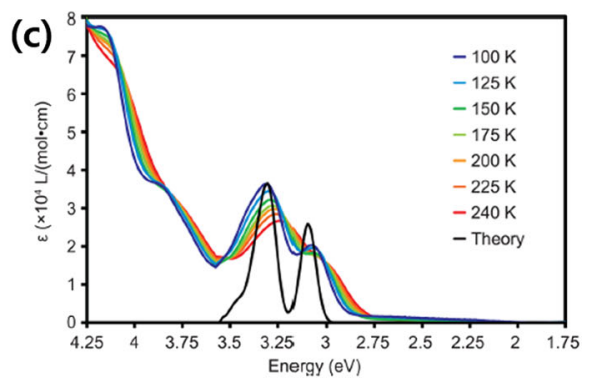

(f)

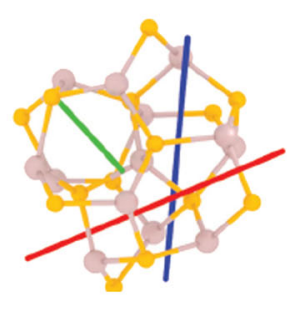

(g)
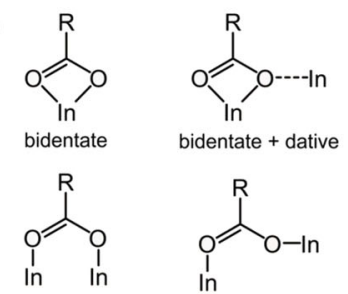

symmetric bridging asymmetric bridging

Fig. 3 Carboxylate Capped InP MSCs with Persistent Absorption Peak at 386 nm. a UV-vis absorption spectra of 386-InP MSCs formed with different reaction temperature. Adapted with permission from ref. ${ }^{35}$. Copyright 2009 American Chemical Society. b Complete single-crystal XRD structure of 386-InP MSC, $\operatorname{In}_{37} \mathrm{P}_{20}\left(\mathrm{O}_{2} \mathrm{CR}\right)_{51}(R=$ Phenylacetate) and $\mathbf{c}$ variable-temperature UV-vis absorption spectra of 386-InP MSCs in the solid state. Adapted with permission from ref. ${ }^{45}$. Copyright 2016 American Chemical Society. $\mathbf{d}$ The $\left[\ln _{21} P_{20}\right]^{3+}$ core of the 386-InP MSC (P: orange, In: purple), e a view of one $\mathrm{InP}$ twistane unit (top) and an excerpt of the cadmoindite unit cell showing structural homology (bottom), and $\mathbf{f}$ a view of the $\ln _{13} \mathrm{P}_{14}$ subunit with T-symmetry derived from the 386-InP MSC illustrating three of twelve interlocking and intersecting polytwistane axes. Adapted with permission from ref. ${ }^{71}$. Copyright 2018 WILEY VCH. $\mathbf{g}$ Ligand binding modes of 386-InP MSC. Of the 51 carboxylate ligands, 12 are identified as bidentate, one is identified as bidentate + dative, five are identified as symmetric bridging, and 33 are identified as asymmetric bridging by SCXRD. Adapted with permission from ref. ${ }^{45}$. Copyright 2016 American Chemical Society.

additional 16 indium atoms bound to surface-exposed phosphorus atoms ${ }^{45}$. The ligand binding modes of the 51 carboxylates also revealed that 12 are bidentate, one is bidentate dative, five are symmetric bridging, and 33 are asymmetric bridging (Fig. $3 \mathrm{~g})^{45}$.

${ }^{13} \mathrm{C}$ NMR and ${ }^{1} \mathrm{H}$ NMR spectroscopies were used to analyze the equilibrium dynamics of surface ligands. Isotopically labeled $\operatorname{In}_{37} \mathrm{P}_{20}\left(\mathrm{O}_{2}{ }^{13} \mathrm{CCH}_{2} \mathrm{Ph}\right)_{51}$ was investigated by NMR, and the interaction between $\operatorname{In}_{37} \mathrm{P}_{20}\left(\mathrm{O}_{2}{ }^{13} \mathrm{CCH}_{2} \mathrm{Ph}\right)_{51}$ and $\mathrm{In}\left(\mathrm{O}_{2}{ }^{13} \mathrm{CCH}_{2} \mathrm{Ph}\right)_{3}$ was traced ${ }^{76}$. The free indium carboxylate in solution is dynamically exchanged with the bound indium carboxylate on the 386-InP MSC, even at room temperature.

Fatty amine ligands have been widely used as surfactants for the synthesis of InP QDs or InP MSCs. When fatty amines such as oleylamine were used along with $\operatorname{In}(\mathrm{Ac})_{3}$ as surfactants/solvents and reacted with (TMS) ${ }_{3} \mathrm{P}$, two kinds of InP MSCs formed (Fig. 4a) ${ }^{12}$, one with the LEET at $\lambda=$ $365 \mathrm{~nm}$ and another with the transition at $\lambda=395 \mathrm{~nm}$. During heating to $170^{\circ} \mathrm{C}, 365 \mathrm{~nm}$ InP MSCs began to form at $45^{\circ} \mathrm{C}$; the optimal $T_{\mathrm{R}}$ was $\sim 70^{\circ} \mathrm{C}$. Application of the Debye-Scherrer formula to the pXRD pattern determined that the $365-\mathrm{nm}$ InP MSCs were $1.1 \mathrm{~nm}$ in size. Further heating from $140^{\circ} \mathrm{C}$ to $170^{\circ} \mathrm{C}$ yielded the formation of $395 \mathrm{~nm}$ InP MSCs (Fig. 4a).
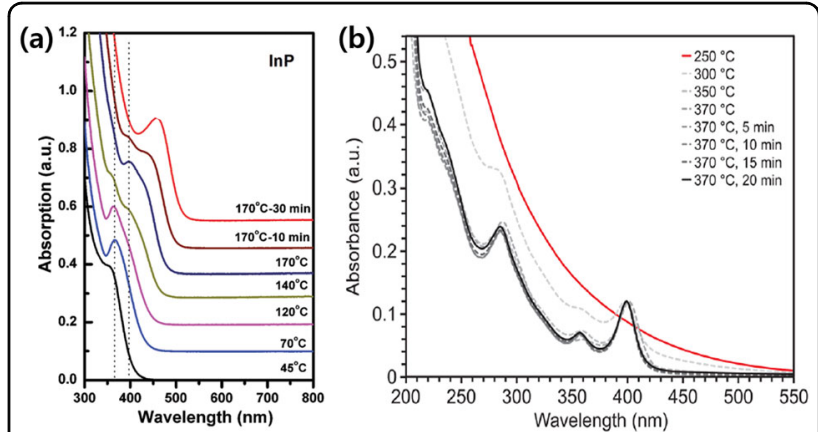

Fig. 4 Oleylamine and octadecylphosphonate capped InP MSCs. a UV-vis absorption of aliquots taken at different temperatures and reaction times during growth of two kinds of amine passivated $\ln P$ MSCs. Adapted with permission from ref. ${ }^{12}$. Copyright 2017 The Royal Society of Chemistry. $\mathbf{b}$ UV-vis absorption of aliquots taken during growth of phosphonate passivated InP MSCs, F397-InP MSCs, as heating up to $370^{\circ} \mathrm{C}$. Adapted with permission from ref. ${ }^{10}$. Copyright 2015 American Chemical Society.

The addition of a fatty amine with carboxylic acid to the $\operatorname{In}(\mathrm{Ac})_{3}$ solution followed by reaction with (TMS) ${ }_{3} \mathrm{P}$ precursors yields various InP MSCs with LEETs at $\lambda>$ $400 \mathrm{~nm}^{35,91}$. Several species of InP MSCs with amine/ carboxylate co-ligands can be synthesized by controlling 
$T_{\mathrm{R}}$ and the amount of amines. When 3-6 equivalents of octylamine relative to the $\operatorname{In}(\mathrm{My})_{3}$ complex were used, $\mathrm{InP}$ MSCs with the LEET at $\lambda=450 \mathrm{~nm}$ appeared at $158 \leq$ $T_{\mathrm{R}} \leq 208^{\circ} \mathrm{C}^{35}$. However, at $T_{\mathrm{R}}=160^{\circ} \mathrm{C}$, excess octylamine with the $\operatorname{In}(\mathrm{My})_{3}$ complex destabilized the MSCs and led to continuous growth of InP QDs ${ }^{10}$.

Adding amines to InP MSCs separately also has an impact $^{91}$. The addition of as little as one equivalent of amine to a 386-InP MSC solution caused a redshift of the absorption peak from $386 \mathrm{~nm}$ to $420 \mathrm{~nm}$. When more than six equivalents of amine were added to the 386-InP MSCs, this redshifted absorption peak at $420 \mathrm{~nm}$ was maintained without a further shift.

The redshifted absorption peak was assumed to be a result of a change in the electronic structure caused by etching of the initial 386-InP MSCs. Reaction of amines with 386-InP MSCs caused etching that yielded crystals of molecules composed of phenylacetate and benzylamine, $\operatorname{In}\left(\mathrm{O}_{2} \mathrm{CH}_{2} \mathrm{C}_{5} \mathrm{H}_{6}\right)_{3}\left(\mathrm{H}_{2} \mathrm{NCH}_{2} \mathrm{C}_{6} \mathrm{H}_{5}\right)_{3}$.

${ }^{31} \mathrm{P}$ NMR spectroscopy revealed selective etching of InP MSCs, even for a small amount of amine reacting with 386-InP MSCs. Especially for InP MSCs, solution-phase ${ }^{31} \mathrm{P}$ NMR is a useful tool for observing the electronic environment of each P in an MSC. 386-InP MSCs showed phosphorus peaks in the chemical shift range between -190 and $-250 \mathrm{ppm}^{10,91}$. When three equivalents of amine were added to a 386-InP MSC solution, a particular peak underwent a large shift in the ${ }^{31} \mathrm{P}$ NMR spectrum ${ }^{92}$. The peak corresponds to the phosphorus that is nearest to the most acidic indium that is etched by three equivalents of amine.

Phosphonic acid is a ligand that binds strongly with cations in semiconductor QDs and MSCs. Octadecylphosphonic acid (ODPA) with $\operatorname{In}(\mathrm{Ac})_{3}$ and (TMS) ${ }_{3} \mathrm{P}$ were used as InP MSC precursors; their reaction in squalane at $370{ }^{\circ} \mathrm{C}$ led to the formation of phosphonate-capped InP $\mathrm{MSCs}^{10}$. ODPA-capped InP MSCs have four distinct absorption peaks at 397, 355, 285, and $238 \mathrm{~nm}$, which correspond to four excitonic transitions (Fig. 4b). The wavelength of the LEET is $397 \mathrm{~nm}$, so they are denoted as F397-InP MSCs. Surprisingly, F397-InP MSCs were stable up to $370^{\circ} \mathrm{C}$ for three days due to the strong binding affinity of phosphonate on the surface indium of the MSCs. The pXRD pattern of F397-InP MSCs showed a zinc-blende structure, and PDF data supported the structural difference from 386-InP MSCs, which form polytwistane ${ }^{10}$.

In summary, carboxylate-passivated 386-InP MSCs, several kinds of amine-passivated InP MSCs, amine/carboxylate co-passivated InP MSCs, and phosphonatepassivated F397-InP MSCs have been synthesized (Table 2). All of the InP MSCs were formed using $\operatorname{In}(\mathrm{Ac})_{3}$ as the indium precursor and (TMS) ${ }_{3} \mathrm{P}$ as the phosphorus precursor; different ligands, such as HMy, oleylamine, and ODPA, for the indium complex led to different types of InP MSCs. Different ligand binding affinities to the MSC surface affect the thermal stability of InP MSCs. Among the InP MSCs, only the structure of the 386-InP MSC has been revealed $\left(\operatorname{In}_{37} \mathrm{P}_{20}\left(\mathrm{O}_{2} \mathrm{CR}\right)_{51}\right)$ by SCXRD. ${ }^{31} \mathrm{P}$ NMR spectroscopy was used to reveal the selective etching of 386-InP MSCs by amines, and pXRD and PDF analysis showed the zinc-blende structure of phosphonate-passivated F397-InP MSCs.

\section{InP MSCs with incorporated heterogeneous atoms}

The incorporation of heterogeneous atoms into semiconductor QDs and MSCs has been widely adopted to modify the optical properties of original nanomaterials or to develop functional materials ${ }^{40,93-97}$. Heterogeneous atoms can be introduced directly at the beginning of the synthesis reaction or indirectly by ion exchange or doping ${ }^{47,98,99}$. For MSCs, the number or position of heterogeneous atoms in their structures can be precisely controlled $^{48,53,100-102}$. In InP nanomaterials, zinc, gallium, cadmium, copper, and tin have been introduced as heterogeneous cations; halides and sulfides have been incorporated as heterogeneous anions to increase the brightness and stability of InP nanomaterials ${ }^{103-110}$. InP MSCs with incorporated heterogeneous atoms have been reported ${ }^{11}$. Here, we consider $\mathrm{Cl}$ and $\mathrm{Zn}$ as heterogeneous atoms in InP MSCs.

\section{InP MSCs with incorporated chlorine}

$\mathrm{Cl}$ has been widely utilized to control the morphology of semiconductor nanomaterials due to the strong binding affinity of $\mathrm{Cl}$ to certain facets during growth ${ }^{109}$. In addition, the introduction of $\mathrm{Cl}$ to the surface of semiconductor nanomaterials by posttreatment enables an increase in the carrier mobility in nanomaterial devices such as field-effect transistors and solar cells ${ }^{111}$.

Two kinds of InP:Cl MSCs were successfully synthesized, which were named F360-InP:Cl MSCs and F399InP:Cl MSCs according to their LEETs (Fig. 5a, b) ${ }^{11}$. The "F" in the MSC names categorizes the group of MSCs that have the same absorption spectrum as the family until a single distinct characteristic is identified. MSCs with incorporated heterogeneous atoms may be heterogeneous as a result of their isomers in both the inorganic crystal structures and surface-binding ligand configurations.

F360-InP:Cl MSCs and F399-InP:Cl MSCs were synthesized using $\operatorname{In}(\mathrm{Ac})_{3}, \mathrm{InCl}_{3}, \mathrm{HMy}$, and $(\mathrm{TMS})_{3} \mathrm{P}$ as molecular precursors. The fate of these types of InP MSCs is determined by $T_{\mathrm{R}}$ and the precursor ratio. F360-InP:Cl MSCs form at low $T_{\mathrm{R}} \sim 50^{\circ} \mathrm{C}$, whereas F399-InP:Cl MSCs grow at a relatively higher temperature of $80 \leq T_{R} \leq$ $110^{\circ} \mathrm{C}$. In addition, F360-InP:Cl MSCs are optimally formed when the amount of $\mathrm{Cl}$ is less than the amount of $\mathrm{HMy}\left(\mathrm{In}(\mathrm{Ac})_{3}: \mathrm{HMy}: \mathrm{InCl}_{3}:(\mathrm{TMS})_{3} \mathrm{P}=1: 3: 0.6: 0.5\right)$, whereas 
Table 2 Kinds of synthesized InP magic-sized clusters.

\begin{tabular}{|c|c|c|c|c|}
\hline & LEET (nm) & Surfactant & Precursor & $\begin{array}{l}\text { Growth temperature } \\
\left({ }^{\circ} \mathrm{C}\right)\end{array}$ \\
\hline \multirow[t]{5}{*}{ InP MSCs } & $386^{45}$ & $\begin{array}{l}\text { Carboxylic acid (from butanoic acid to stearic acid, phenylacetic } \\
\text { acid, or oleic acid) }\end{array}$ & $\ln (\mathrm{Ac})_{3},(\mathrm{TMS})_{3} \mathrm{P}$ & 110 \\
\hline & $365^{12}$ & Alkylamine (oleylamine) & $\ln (\mathrm{AC})_{3},(\mathrm{TMS})_{3} \mathrm{P}$ & 70 \\
\hline & $395^{12}$ & Alkylamine (oleylamine) & $\ln (\mathrm{AC})_{3},(\mathrm{TMS})_{3} \mathrm{P}$ & $140-170$ \\
\hline & $450^{12,35}$ & Carboxylic acid (myristic acid), alkylamine (octylamine) & $\ln (\mathrm{AC})_{3,}(\mathrm{TMS})_{3} \mathrm{P}$ & 158 \\
\hline & $397^{10,12}$ & Phosphonic acid (octadecylphosphonic acid) & $\ln (\mathrm{AC})_{3,}(\mathrm{TMS})_{3} \mathrm{P}$ & 370 \\
\hline \multirow[t]{4}{*}{ InP:CI MSCs } & $360^{11,12}$ & $\begin{array}{l}\text { Halogen ( } \mathrm{InCl}_{3} \text { or TMS-Cl) and carboxylic acid (myristic acid or } \\
\text { oleic acid) }\end{array}$ & $\ln (\mathrm{AC})_{3},\left.\ln C\right|_{3},(\mathrm{TMS})_{3} \mathrm{P}$ & $25-80$ \\
\hline & & & 386-InP MSC, InCl 3 & \\
\hline & $399^{11}$ & Halogen $\left(\mathrm{InCl}_{3}\right)$ and carboxylic acid (HMy or oleic acid) & $\ln (\mathrm{AC})_{3}, \ln \mathrm{Il}_{3},(\mathrm{TMS})_{3} \mathrm{P}$ & $80-110$ \\
\hline & & & $\mathrm{F} 360-\ln \mathrm{P}: \mathrm{Cl} \mathrm{MSC}, \mathrm{InCl}_{3}$ & \\
\hline \multirow[t]{5}{*}{ InP:Zn MSCs } & $360^{11}$ & $\begin{array}{l}\text { Phosphonic acid (octadecylphosphonic acid) and carboxylate } \\
\text { (stearate or oleate) }\end{array}$ & $\begin{array}{l}\ln (\mathrm{AC})_{3}, \mathrm{Zn}(\mathrm{St})_{2} \text { or } \mathrm{Zn}(\mathrm{Ol})_{2} \\
(\mathrm{TMS})_{3} \mathrm{P}\end{array}$ & 180 \\
\hline & $408^{11}$ & $\begin{array}{l}\text { Phosphonic acid (octadecylphosphonic acid) and carboxylate } \\
\text { (stearate or oleate) }\end{array}$ & $\begin{array}{l}\ln (\mathrm{AC})_{3}, \mathrm{Zn}(\mathrm{St})_{2} \text { or } \mathrm{Zn}(\mathrm{Ol})_{2} \\
(\mathrm{TMS})_{3} \mathrm{P}\end{array}$ & 300 \\
\hline & & & F360-InP:Zn MSC & \\
\hline & $393^{11}$ & Phosphonic acid (octadecylphosphonic acid) & $\begin{array}{l}\ln (\mathrm{AC})_{3}, \mathrm{Zn}(\mathrm{St})_{2} \text { or } \mathrm{Zn}(\mathrm{Ol})_{2} \\
(\mathrm{TMS})_{3} \mathrm{P}\end{array}$ & 300 \\
\hline & & & F408-InP:Zn MSC & \\
\hline
\end{tabular}

$\ln (A C)_{3}$ indium acetate, $(T M S)_{3} P$ tris(trimethylsilyl)phosphine, $I n C l_{3}$ indium chloride, $T M S-C l$ trimethylsilyl chloride, $\mathrm{Zn}(\mathrm{St})_{2}$ zinc stearate, $\mathrm{Zn}(\mathrm{Ol})_{2}$ zinc oleate.

F399-InP:Cl MSCs are synthesized with the highest yield when the amount of $\mathrm{Cl}$ is high $\left(\operatorname{In}(\mathrm{Ac})_{3}: \mathrm{HMy}_{\mathrm{InCl}}\right.$ : $\left.(\mathrm{TMS})_{3} \mathrm{P}=1: 3: 1: 0.5\right)$.

Interestingly, two InP MSCs were synthesized by the other route, i.e. the conversion process. 386-InP MSCs that did not have $\mathrm{Cl}$ were used as well-defined starting scaffolds for conversion synthesis. Reaction of assynthesized 386-InP MSCs and 56 equivalents of $\mathrm{InCl}_{3}$ yielded F360-InP:Cl MSCs (Fig. 5c). The conversion mechanism of F360-InP:Cl MSCs from 386-InP MSCs was revealed to be etching by $\mathrm{HCl}$ in situ generated by the reaction of $\mathrm{InCl}_{3}$ and remaining carboxylic acid. A proton-coupled ${ }^{31} \mathrm{P}$ NMR spectrum showed that the etching process released $\mathrm{PH}_{3}$. The conversion reaction also occurred when $\mathrm{HCl}$ was added directly to the 386-InP MSC solution. However, the isosbestic point of the absorption spectrum was not visible during the etching reaction from 386-InP MSCs to F360-InP:Cl MSCs; this absence indicates that the reaction is not one-to-one but involves intermediate species such as fragments of MSCs. The concept of fragmentation of MSCs and fragment reassembly was recently proposed ${ }^{47,112,113}$.

F399-InP:Cl MSCs were also synthesized by a conversion route (Fig. 5d). When F360-InP:Cl MSCs were heated to a temperature $>110{ }^{\circ} \mathrm{C}$, the absorption spectrum of
F360-InP:Cl MSCs disappeared completely, and a sharp absorption peak emerged at $399 \mathrm{~nm}$. These changes can be understood to represent thermal fragmentation of F360-InP:Cl MSCs and reassembly from fragments. pXRD and Raman spectra showed that F399-InP:Cl MSCs have a zinc-blende structure, whereas 386-InP MSCs and F360InP MSCs are polytwistanes; i.e., the structure evolved as conversion proceeded from 386-InP MSCs to F360-InP MSCs to F399-InP MSCs.

MSCs may be expected to have characteristics that are intermediate between those of molecules and nanocrystals. Depending on their sizes or structures, MSCs might have physical and chemical properties that resemble those of a molecular system or a nanocrystal system. For example, the transition from molecular vibrations to nanocrystal phonons was investigated for a series of three CdSe $\mathrm{MSCs}^{66}$. Phonon confinement and molecular vibration differ in how their vibrational peaks depend on temperature. Variable-temperature Raman spectra of three CdSe MSCs, $\mathrm{Cd}_{35} \mathrm{Se}_{20} \mathrm{X}_{30} \mathrm{~L}_{30}, \mathrm{Cd}_{56} \mathrm{Se}_{35} \mathrm{X}_{42} \mathrm{~L}_{42}$, and $\mathrm{Cd}_{84} \mathrm{Se}_{56} \mathrm{X}_{56} \mathrm{~L}_{56}$ MSCs (X: benzoate, L: butylamine), revealed a transition of MSCs from molecular characteristics to nanocrystal characteristics.

The transition from a molecular system to a nanocrystal system was investigated for 386-InP MSCs, F360-InP:Cl 

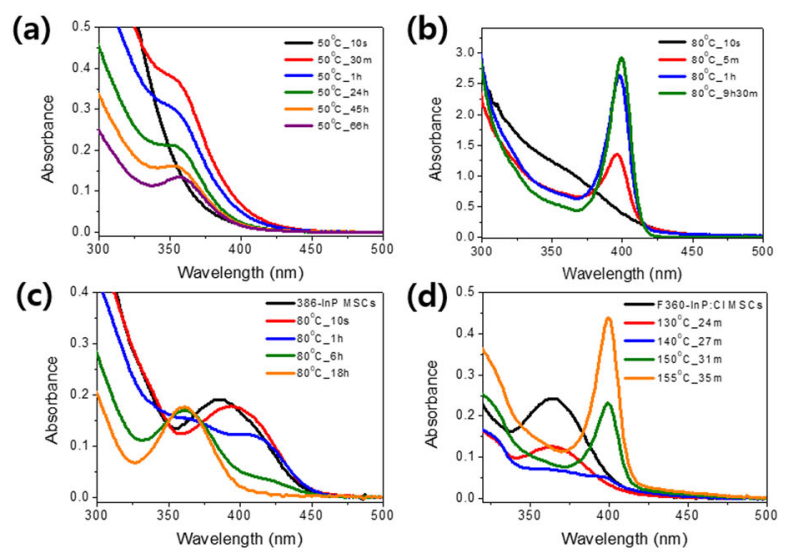

(e)

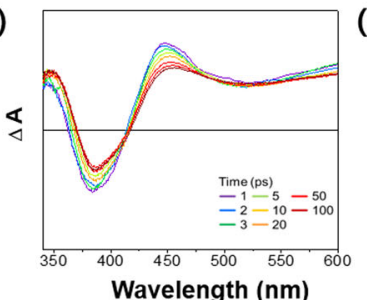

(f)

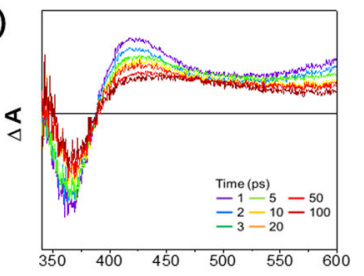

Wavelength $(\mathrm{nm})$

(g)
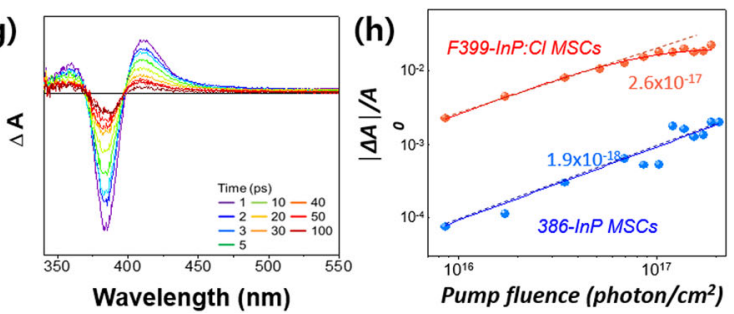

(i)

$\ln \left(\mathrm{OOCR}_{1}\right)_{3}, \mathrm{R}_{2} \mathrm{COOH}, \mathrm{P}\left(\mathrm{SiMe}_{3}\right)_{3}$



Conversion Synthesis

Direct Synthesis $\ln \mathrm{Cl}_{3,}, \ln \left(\mathrm{OOCR}_{1}\right)_{3}$ $\mathrm{R}_{2} \mathrm{COOH}, \mathrm{P}\left(\mathrm{SiMe}_{3}\right)_{3}$ $\mathrm{R}_{1}=\mathrm{CH}_{3}, \mathrm{R}_{2}=\mathrm{C}_{13} \mathrm{H}_{27}$

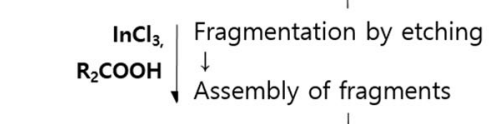

年

$80^{\circ} \mathrm{C}$

$\mathrm{InCl}_{3}$ Thermal fragmentation Heat $\downarrow$

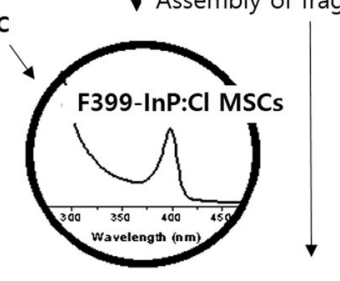

Fig. 5 InP MSCs with incorporated chlorine atoms. UV-vis absorption spectra of aliquots taken over time during (a) direct synthesis of F360-InP:Cl MSCs from indium acetate, $\mathrm{HMy}_{1} \mathrm{InCl}_{3}$ and $(\mathrm{TMS})_{3} \mathrm{P}$ at $50{ }^{\circ} \mathrm{C}$, (b) direct synthesis of $\mathrm{F} 399-\operatorname{InP}: \mathrm{Cl}$ MSCs from indium acetate, InCl ${ }_{3}, \mathrm{HMy}$ and $(\mathrm{TMS})_{3} \mathrm{P}$ at $80^{\circ} \mathrm{C}$, (c) conversion synthesis of F360-InP:CI MSCs from as-synthesized 386-MA InP MSCs using 56 equivalents of InCl 3 at $80{ }^{\circ} \mathrm{C}$, and $(\mathbf{d})$ conversion synthesis of F399-InP:Cl MSCs from F360-InP:Cl MSCs by heating. TA spectra of e 386-InP MSCs, f F360-InP:Cl MSCs, and $\mathbf{g}$ F399-InP:Cl MSCs. h Plot for normalized LEET bleach signals of 386-InP MSCS and F399-InP:CI MSCS with the estimated absorption cross section $\sigma_{a b s}\left(\mathrm{~cm}_{-1}{ }^{1}\right)$. i Schematic illustration showing direct or conversion synthesis pathway for Cl-incorporated InP MSCs. Adapted with permission from ref. ${ }^{11}$. Copyright 2019 NATURE PUBLISHING GROUP.

MSCs and F399-InP:Cl MSCs ${ }^{11}$. The TA spectra of F399InP:Cl MSCs were significantly distinct from those of 386InP MSCs and F360-InP:Cl MSCs (Fig. 5e-g). The TA spectra of 386-InP MSCs and F360-InP:Cl MSCs showed similar spectral features, such as a broad and long-lived photoinduced absorption signal in the lower-energy region of band-edge bleaching around 386 and $360 \mathrm{~nm}$, respectively (Fig. 5e, f). The significantly broad and long-lived signals are attributable to the transition from the lowest excited electronic state to higher excited electronic states, which is typically observed in molecular systems ${ }^{114}$. The TA spectra of F399-InP:Cl MSCs showed strong band-edge bleaching with a narrow photoinduced absorption signal in the low-energy region (Fig. 5g); this signal is typical of the TA spectral features observed in well-defined QDs and describes the relaxation dynamics of excitons in quantumconfined systems ${ }^{115-117}$. The absorption cross section at the LEET was 14 times larger in F399-InP:Cl MSCs than in the 386-InP MSC counterparts (Fig. 5h). This difference indicates that their different electronic structures lead to a distinct difference in the absorption cross section as well as their optical properties. Information about the size and structure suggests that large F399-InP:Cl MSCs should evolve toward a zinc-blende structure close to the bulk thermodynamic equilibrium that has high symmetry; i.e., evolution of the character from molecule-like to QD-like was observed for the series from 386-InP MSCs to F360InP:Cl MSCs and F399-InP:Cl MSCs (Fig. 5i).

\section{InP MSCs with incorporated zinc}

$\mathrm{Zn}$ is important for InP QDs and InP MSCs because bright InP-based QDs typically have Zn-chalcogenide shell layers (e.g., $\mathrm{ZnSe}, \mathrm{ZnS}$ ). Alloy formation at core-shell interfaces (InZnSe; InZnS) and interdiffusion of $\mathrm{Zn}$ into InP 
cores strongly affect the emission properties of $\mathrm{QDs}^{104,118,119}$. Similar to the series of InP:Cl MSCs, InP:Zn MSCs can be synthesized from molecular precursors or by a conversion method ${ }^{11}$. Three kinds of InP:Zn MSCs, F360InP:Zn MSCs, F408-InP:Zn MSCs, and F393-InP:Zn MSCs (Fig. 6a, b), were produced by heating a reaction solution containing an In-ODPA complex, zinc stearate $\left(\mathrm{Zn}(\mathrm{St})_{2}\right)$, and (TMS) $)_{3} \mathrm{P}$ at $T_{\mathrm{R}}$ up to $300^{\circ} \mathrm{C}$. Selective synthesis of pure InP:Zn MSC species can be achieved by controlling $T_{\mathrm{R}}$ and the amount of ODPA. F360-InP:Zn MSCs were synthesized at $180^{\circ} \mathrm{C}$ using twice the amount of ODPA relative to indium (Fig. 6a), with fixed amounts of the other precursors at In:Zn:(TMS) ${ }_{3} \mathrm{P}=1: 1: 0.5$ for all kinds of InP:Zn MSCs. Before crystallization, ODPA has high binding affinity to the indium cation, so a high ODPA content relative to indium slows the reaction rate. The growth temperature was $300^{\circ} \mathrm{C}$ for both F408-InP:Zn MSCs and F393-InP:Zn MSCs, but 1.5 times and 1.85 times ODPA relative to indium, respectively, were used. F360-InP:Zn MSCs grew under the slowest reaction conditions.
When the $T_{\mathrm{R}}$ of the F360-InP:Zn MSC solution was increased to $300^{\circ} \mathrm{C}$, F408-InP:Zn MSCs grew by a conversion mechanism. Further heating at $300^{\circ} \mathrm{C}$ for $24 \mathrm{~h}$ converted F408-InP:Zn MSCs to F393-InP:Zn MSCs (Fig. 6b). The evolution of the UV-vis absorption spectra was quantized during the conversion of F360-InP:Zn MSCs to F408-InP:Zn MSCs, whereas the evolution of the UV-vis absorption spectra was continuous during the conversion of F408-InP:Zn MSCs to F393-InP:Zn MSCs; the process showed a continuous blueshift from $393 \mathrm{~nm}$ to $408 \mathrm{~nm}$. ${ }^{1} \mathrm{H}$ NMR and FT-IR data revealed the conversion of F408-InP: Zn MSCs to F393-InP:Zn MSCs as the surface ligand was changed. ODPA and stearate were used with In, Zn, and P precursors, so two kinds of ligand could attach to the surface of InP:Zn MSCs. F408-InP:Zn MSCs have both of these ligands, with a 5.9:1 phosphonate:carboxylate ratio. As conversion proceeded, carboxylate ligands were removed from the surface of F408-InP:Zn MSCs, followed by the formation of F393-InP:Zn MSCs, which were passivated by only phosphonate ligands.
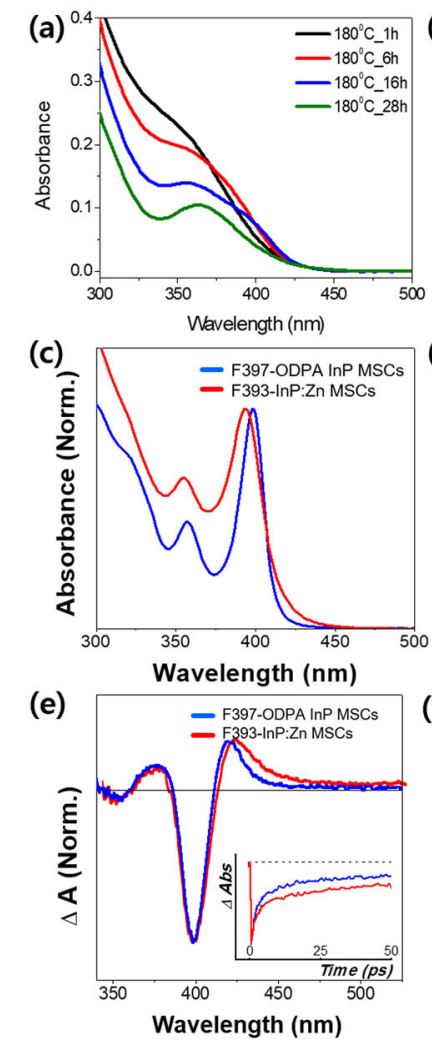

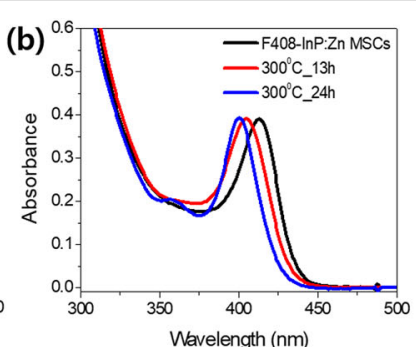

(d)
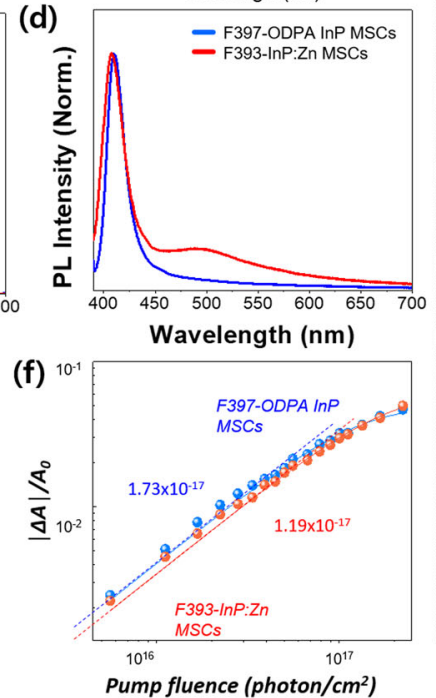

(g)

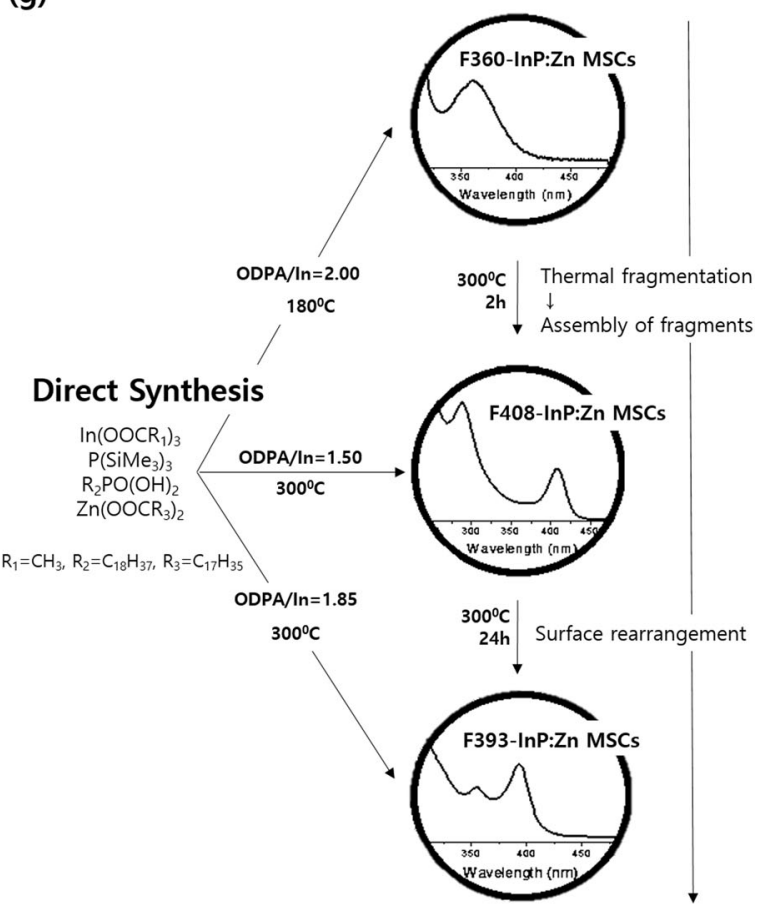

Fig. 6 InP MSCs with incorporated zinc atoms. UV-vis absorption spectra of aliquots taken during direct synthesis of F360-InP:Zn MSCs from In $(\mathrm{Ac})_{3}, \mathrm{ODPA},(\mathrm{TMS})_{3} \mathrm{P}$ and $\mathrm{Zn}(\mathrm{St})_{2}$ precursors at $180{ }^{\circ} \mathrm{C}$ and $(\mathbf{b})$ conversion synthesis of F393-InP:Zn MSCs from as-synthesized F408-InP:Zn MSCs at $300^{\circ} \mathrm{C}$. $\mathbf{c}$ Steady-state absorption and $\mathbf{d}$ PL spectra of F397-ODPA InP MSCs and F393-InP:Zn MSCs. e Normalized TA spectra at 2 ps of F397-ODPA InP MSCs and F393-InP:Zn MSCs. (Inset: normalized TA decay profile of F397-ODPA InP MSCs at 397 nm and of F393-InP:Zn MSCs at 393 nm). f Plot for normalized LEET bleach signals for F397-ODPA InP MSCs and F393-InP:Zn MSCs with the estimated absorption cross section $\sigma_{\text {abs }}\left(\mathrm{cm}^{-1}\right)$. $\mathbf{g}$ Schematic illustration showing direct or conversion synthesis pathway for Zn-incorporated InP MSCs. Adapted with permission from ref. ${ }^{11}$. Copyright 2019 Nature Publishing Group. 
$\mathrm{Zn}$ incorporation affects several optical spectra of InP $\mathrm{MCSs}^{11}$. Among the series of InP:Zn MSCs, F393-InP:Zn MSCs are solely passivated by phosphonate ligands, so they can be compared with F397-InP MSCs that are capped with phosphonate ligands. In the steady-state absorption spectra, F397-ODPA InP MSCs showed a very narrow LEET at $397 \mathrm{~nm}$ with a second electronic transition at $356 \mathrm{~nm}$, whereas F393-InP:Zn MSCs showed a slightly broader and blueshifted LEET at $393 \mathrm{~nm}$ with a second transition at $354 \mathrm{~nm}$ (Fig. 6c) ${ }^{11}$. The blueshifted absorption spectrum of F393-InP:Zn MSCs indicates a decreased effective size of the quantum-confined core; i.e. $\mathrm{Zn}$ cations might be mostly incorporated at the surface of the MSCs.

In the PL emission spectra, F393-InP:Zn MSCs had a brighter and broader LEET than F397-ODPA InP MSCs $(\text { Fig. } 6 \mathrm{~d})^{11}$. The emissive features suggest that the incorporated $\mathrm{Zn}$ cations facilitate carrier trapping and intensify the trap emission in F393-InP:Zn MSCs.

The TA spectra of F397-ODPA InP MSCs and F393InP:Zn MSCs are strikingly similar, with clear spectral features that arise from the exciton relaxation dynamics in quantum-confined systems (Fig. 6e) ${ }^{11}$. F393-InP:Zn MSCs had a broader photoinduced absorption signal in a lowerenergy region of band-edge bleaching with a longer-lived TA decay profile compared to F397-ODPA InP MSCs. These changes are consistent with the increase in the density of trap states after the incorporation of $\mathrm{Zn}$. This incorporation could reduce the symmetry of F393-InP:Zn MSCs and thereby reduce the absorption cross section and widen the absorption and emission spectral features (Fig. 6f). To summarize, the series of InP:Zn MSCs undergo conversion processes similar to the series of InP: $\mathrm{Cl}$ MSCs (Fig. 6g), and incorporation of $\mathrm{Zn}$ reduces the symmetry of InP MSCs to yield trap-induced optical properties.

\section{Applications of InP MSCs}

Using InP MSCs as precursors for QDs and understanding the MSC-mediated growth mechanism

Research on intermediates such as MSCs has revealed the growth process of QDs and provides the possibility of controlling intermediate species. Isolated and characterized InP MSCs have been used as synthesis precursors for InP QDs and nonspherical InP NSs. InP QDs have been synthesized using 386-InP MSCs as precursors ${ }^{10,76,120}$. The size distribution and sharpness of the excitonic absorption peak of InP QDs synthesized from 386-InP MSCs are superior to those of conventional InP QDs synthesized from molecular precursors ${ }^{120}$.

In the synthesis strategy, the size of InP QDs is determined by controlling the concentration of 386-InP MSC precursors $^{10}$. The size of the InP QDs increases as the concentration of 386-InP MSC precursors is increased.
Representative MSC-mediated growth mechanisms have been divided into two types: (1) monomer-driven growth and (2) MSC assembly ${ }^{121}$. Monomer-driven growth can occur by monomer deposition onto MSC seeds, quantized cluster growth, or complete dissolution/ renucleation for each MSC population. MSC assembly occurs by attachment among MSCs.

386-InP MSCs showed both of these growth pathways to form QDs depending on the additives and $T_{\mathrm{R}}{ }^{71,76}$. When 386-InP MSCs were reacted with HMy or $\operatorname{In}(\mathrm{My})_{3}$ as additives, the yields of grown $\mathrm{InP}$ nanoparticles (NPs) were very different at $T_{\mathrm{R}}=130,150,200,250$, and $300^{\circ} \mathrm{C}$ (Fig. 7a). At $T_{\mathrm{R}}=130^{\circ} \mathrm{C}$, only 386 -InP MSCs dissolved. Surprisingly, NPs did not grow after the dissolution of MSCs, even if $T_{\mathrm{R}}$ was increased. This result indicates that the dissolution of 386-InP MSCs does not provide a monomer reservoir for nucleation (off-path decomposition). At $T_{\mathrm{R}}=150^{\circ} \mathrm{C}$, agglomerates that had no particular shape or size formed, and the chemical yield was low (Fig. 7b). The agglomerates might originate from the assembly of 386-InP MSCs or their fragments, and the low chemical yield may be a result of competition with off-path decomposition of 386-InP MSCs. However, $T_{\mathrm{R}}>$ $200^{\circ} \mathrm{C}$ yielded uniform spherical InP QDs at high yield as a result of complete dissolution/renucleation of 386-InP MSCs (Fig. 7c).

386-InP MSC conversion to InP QDs depending on $T_{\mathrm{R}}$ may be a result of competing pathways (Fig. 7d) ${ }^{76}$. As $T_{\mathrm{R}}$ is increased, 386-InP MSCs convert to monomers and fragments before the growth of InP QDs. The source of InP QDs could be monomers, fragments, and other monomer species generated from fragments. If $T_{\mathrm{R}}$ is high $\left(>200^{\circ} \mathrm{C}\right)$ enough to almost produce monomers, then monomer-driven InP QDs grow; otherwise, fragments of 386-InP MSCs participate in growth to produce agglomerates. At low $T_{R}$, off-path decomposition of 386-InP MSCs is predominant.

The use of additives with MSC precursors that include carboxylate leads to fast dissolution of 386-InP MSCs followed by monomer-driven growth of InP QDs ${ }^{76}$. When 386-InP MSCs were reacted with (TMS) $)_{3} \mathrm{P}$ additives at $T_{\mathrm{R}}<125^{\circ} \mathrm{C}$, spherical-shaped InP QDs grew by assembly of MSCs. Due to the 386-InP MSC assemblies, the grown InP QDs retain the polytwistane phase of the initial structure (Fig. 7f). Several spectra indicate that (TMS) ${ }_{3} \mathrm{P}$ drives the surface decomposition of 386-InP MSCs to yield MSC assemblies (Fig. 7g). 386-InP MSCs do not dissolve entirely, so their core-dominated structural properties could remain.

\section{Using InP MSCs as precursors to achieve diverse morphology InP nanostructures}

InP MSCs have also been used as precursors to control the morphology of InP NSs. Branched nanostructures 


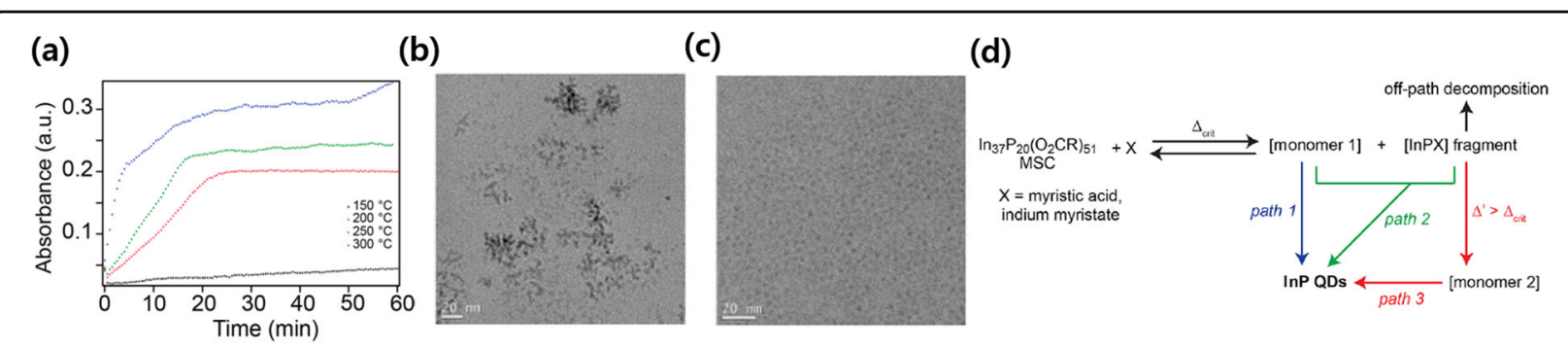

(e)

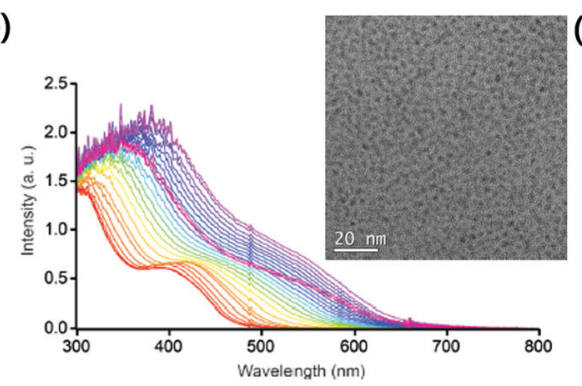

(f)

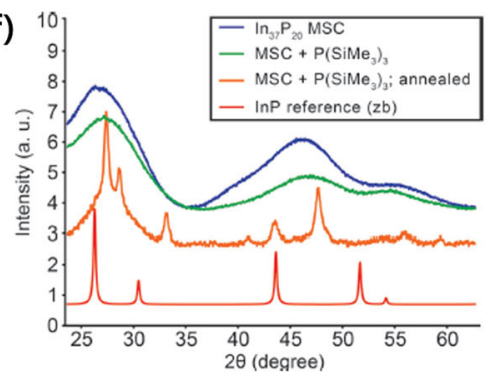

(g)

$$
\begin{gathered}
\ln _{37} \mathrm{P}_{20}\left(\mathrm{O}_{2} \mathrm{CR}\right)_{51}+2 \mathrm{P}\left(\mathrm{SiMe}_{3}\right)_{3} \\
\downarrow \\
\ln _{29} \mathrm{P}_{14}\left(\mathrm{O}_{2} \mathrm{CR}\right)_{45}+6 \mathrm{Me}_{3} \mathrm{SiO}_{2} \mathrm{CR}+8[\mathrm{InP}]
\end{gathered}
$$

Fig. 7 Mechanism studies on growth from InP MSCs to InP NPs. a Intensity change at 500 nm during the evolution of $386-I n P$ MSC at different temperature. TEM images showing (b) agglomerates synthesized from MSCs at $150^{\circ} \mathrm{C}$ and (c) spherical particles synthesized from MSCs at $250^{\circ} \mathrm{C}$. d Proposed competing pathways for MSC conversion to InP QDs dependent on temperature and additives. Adapted with permission from ref. ${ }^{76}$. Copyright 2019 American Chemical Society. e In situ UV-vis spectra of the reaction between 386-InP MSC and 23 equivalents of (TMS) 3 P injected over $1 \mathrm{~h}$ at $100^{\circ} \mathrm{C}$. (Inset: TEM image of synthesized InP NPS). $\mathbf{f}$ XRD patterns of the 386-InP MSCs, synthesized InP NPs from the reaction between 386-InP MSC and 23 equivalents of (TMS) $)_{3}$ injected over $1 \mathrm{~h}$ at $100{ }^{\circ} \mathrm{C}$, annealed synthesized InP NPs and zinc-blende InP reference. $\mathbf{g} \mathrm{A}$ proposed reaction between the 386-InP MSCS and (TMS) ${ }_{3}$ P. Adapted with permission from ref. ${ }^{71}$. Copyright 2018 WILEY VCH.

(BNSs) and hyperbranched nanostructures (HBNSs)/ dendrimer-like nanostructures (DLNSs) have been synthesized (Fig. 8) using 386-InP MSCs and molecular additives as precursors ${ }^{80}$. At $T_{\mathrm{R}}=300{ }^{\circ} \mathrm{C}$, without additives, 386-InP MSCs grew into spherical InP QDs (Fig. 7c), whereas with $\operatorname{In}(\mathrm{My})_{3}$ or $\operatorname{In}(\mathrm{Ac})_{3}$ molecular additives, 386-InP MSCs grew to form InP BNSs or InP HBNSs/DLNSs (Fig. 8).

To understand how MSCs mediate the shape evolution, the process of InP BNS growth from 386-InP MSCs was investigated. Upon heating, 386-InP MSCs fragmented before further evolution. Fragmentation was significantly impeded by extra indium complexes such as $\operatorname{In}(\mathrm{My})_{3}$ but accelerated by extra fatty acids such as HMy. Co-use of extra $\operatorname{In}(\mathrm{My})_{3}$ and HMy with 386-InP MSCs resulted in InP BNSs (Fig. 8a, b). InP BNSs may grow from 386-InP MSCs when the concentration of monomers released by MSC fragmentation reaches a critical concentration for nucleation; the new nuclei may grow rapidly to form tetrahedral seeds and further to form BNSs while consuming monomers and fragments, possibly via oriented attachment ${ }^{80}$.

When $\operatorname{In}(\mathrm{Ac})_{3}$ was used instead of $\operatorname{In}(\mathrm{My})_{3}$ as an additive for the reaction with 386-InP MSCs, BNSs aggregated to form HBNSs/DLNSs (Fig. 8c) ${ }^{80}$. The InP HBNS/DLNS has a three-dimensional morphology with many branches, and its size can be controlled in the range of $20-30 \mathrm{~nm}$ by changing the total concentration of precursors. Initially formed BNSs partially capped by short acetate ligands interact with each other via the van der Waals force.

In syntheses of anisotropic chalcogenide NSs, structuredirecting agents are commonly used ${ }^{78,79,122}$. The synthesis of InP BNSs and InP HBNS/DLNSs does not require such structure-directing agents. This difference demonstrates that sophisticated control of the growth pathways offers NSs that cannot be attained using conventional synthetic protocols.

\section{Developing the luminescent property of InP MSCs}

Semiconductor MSCs have luminescent properties and therefore have applications in luminous devices such as LEDs $^{82,87}$. To develop the luminescent property of InP MSCs, amine-passivated InP MSCs with LEET $=365 \mathrm{~nm}$ have been used as the core to synthesize shell layers ${ }^{12}$.

The use of a $\mathrm{ZnS}$ shell overcoating to improve the luminescent property of InP MSCs achieved only 4\% QY (Fig. 9a $)^{12}$. The QY after shelling may be low because the HOMO-LUMO levels of $365 \mathrm{~nm}$ InP MSCs are positioned at high energies due to the extremely small size, so exciton confinement to the core by a wide-bandgap shell is difficult. When five layers of $\mathrm{ZnS}$ were overcoated on $365 \mathrm{~nm}$ InP MSCs, a redshifted PL peak of $480 \mathrm{~nm}$ 
(a)

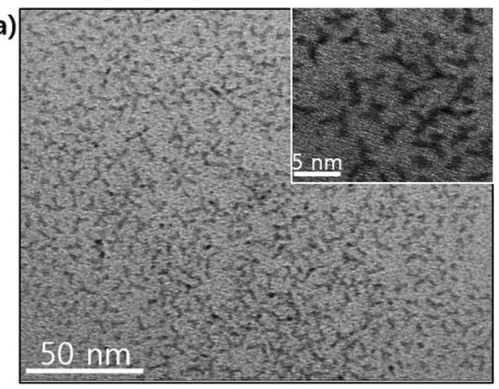

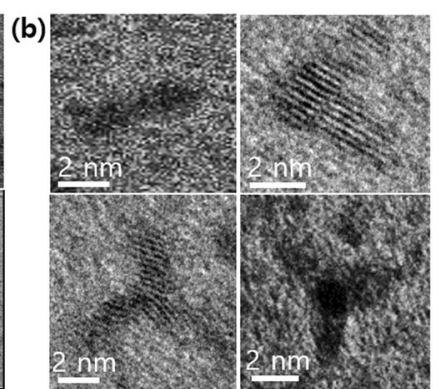

(c)

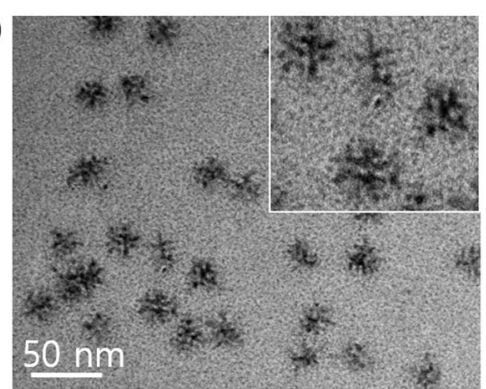

Fig. 8 Controlling morphology of InP NPs using InP MSCs as precursors. TEM images of $(\mathbf{a}, \mathbf{b})$ InP BNSs, which were synthesized by the reaction of 386-InP MSCs with In(My) $)_{3}$ at $220^{\circ} \mathrm{C}$, c InP HBNSs/DLNSs which were synthesized by the reaction of 386-InP MSCs with In $(\mathrm{Ac})_{3}$ at $220^{\circ} \mathrm{C}$. Adapted with permission from ref. 80. Copyright 2019 The Royal Society of Chemistry.

emerged from nondetectable band-edge emission (Fig. 9b, black line). The large redshift was explained as exciton movement to the $\mathrm{ZnS}$ shell from the large bandgap InP MSCs. When 20 layers of $\mathrm{ZnS}$ were deposited, a new emission peak at $560 \mathrm{~nm}$ corresponding to interfacial traps appeared without a further increase in the QY (Fig. 9b, red line). The PL lifetime was measured for the emission at $480 \mathrm{~nm}$ and $560 \mathrm{~nm}$ (Fig. 9c). When five layers of $\mathrm{ZnS}$ were coated on the InP MSCs, the PL lifetime was $34.2 \mathrm{~ns}$ at $480 \mathrm{~nm}$. When 20 layers of $\mathrm{ZnS}$ were used, the PL lifetime was $62.3 \mathrm{~ns}$ at $480 \mathrm{~nm}$ and $248.5 \mathrm{~ns}$ at $560 \mathrm{~nm}$. The extension of the lifetime is consistent with trap emission profiles originating from interfacial traps or the outer surface of the InP/ZnS MSCs.

\section{Conclusion and perspective}

This paper has reviewed the history of InP QD synthesis and research on InP MSCs, which were identified as intermediates during the growth of InP QDs. We classified the research on InP MSCs into two parts: (1) synthesis and characterization and (2) applications. InP MSCs passivated with carboxylate, amine, or phosphonate ligands or that incorporate heterogeneous atoms $(\mathrm{Cl}$ or $\mathrm{Zn}$ ) were synthesized by lowering $T_{\mathrm{R}}$ or introducing strongly bound ligands such as phosphonic acid. Among InP MSCs, the structure of 386-InP MSCs has been shown to be $\operatorname{In}_{37} \mathrm{P}_{20}\left(\mathrm{O}_{2} \mathrm{CR}\right)_{51}$ with a polytwistane phase. This knowledge of the structure accelerated the study of the intermediate characteristics of MSCs that are at a size near the boundary between molecules and QDs. TA characterization successfully revealed the optical evolution from a unimolecular to colloidal-QD-like character in the InP:Cl MSC or InP:Zn MSC series.

InP MSCs have been used as synthesis precursors for high-quality InP QDs or diverse morphology InP NSs. Elongated InP BNSs, HBNSs/DLNSs, and spherical QDs have been successfully formed from InP MSCs by using MSC-mediated growth mechanisms such as monomerdriven growth or MSC assembly. Future research on InP (a)
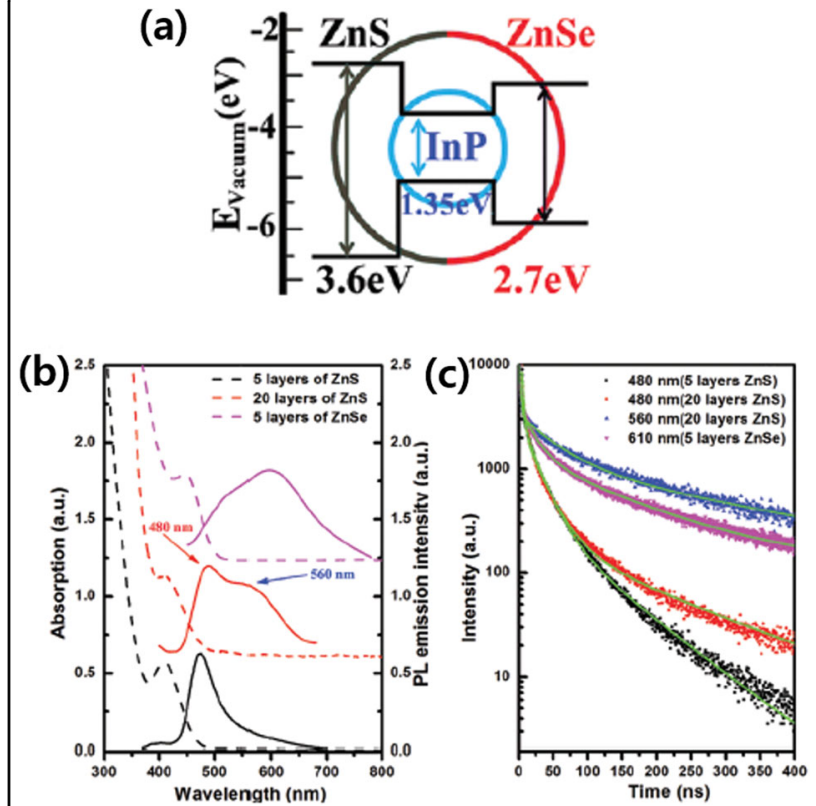

Fig. 9 Developing the luminescent property of InP MSCs. a Potential energy diagram for InP, ZnS, and ZnSe bulk materials. b UV-vis absorption, PL spectra, and (c) PL lifetime of ZnS coated InP MSCs. Adapted with permission from ref. ${ }^{12}$. Copyright 2017 The Royal Society of Chemistry.

MSCs could use various kinds of InP MSCs as precursors to further develop homogeneous or functional nanomaterials. Until now, research has been limited to experiments that used 386-InP MSCs as precursors. Various InP MSCs differ in their structure, thermal stability, and ligands; the development of methods such as preservation/ dissolution or attachment/detachment in the reaction environment to control InP MSCs might allow the synthesis of high-quality and functional InP NSs. The information gained might enable a further understanding of the nucleation and growth mechanism of III-V compound nanocrystal systems. 
Finally, isomerization of II-VI semiconductor MSCs has been recently observed ${ }^{50}$. Reversible structural isomerization in the MSC system bridges the difference between molecules and nanocrystals. We expect isomerization phenomena in III-V semiconductor MSCs and the existence of a difference between III-V compound semiconductor MSCs and II-VI compound semiconductor MSCs. The two groups have atomically precise structures, so the fundamental nature of the ionic and covalent characters in the lattice could also be clearly defined.

\section{Reporting summary}

Further information on research design is available in the Nature Research Reporting Summary linked to this article.

\section{Acknowledgements}

This research was supported by the Creative Materials Discovery Program through the National Research Foundation of Korea (NRF) funded by the Ministry of Science and ICT (No. 2019M3D1A1078302), by an NRF grant funded by the Korean government (MSIT) (No. NRF-2019R1A2C2084013), and by an NRF grant funded by MSIT (No. 2020R1A5A1019141).

\section{Conflict of interest}

The authors declare no competing interests.

\section{Publisher's note}

Springer Nature remains neutral with regard to jurisdictional claims in published maps and institutional affiliations.

Supplementary information The online version contains supplementary material available at https://doi.org/10.1038/s41427-021-00300-4.

Received: 22 October 2020 Revised: 10 February 2021 Accepted: 15 February 2021.

Published online: 23 April 2021

\section{References}

1. Smith, A. M. \& Nie, S. Semiconductor nanocrystals: structure, properties, and band gap engineering. Acc. Chem. Res. 43, 190-200 (2010).

2. Medintz, I. L., Uyeda, H. T., Goldman, E. R. \& Mattoussi, H. Quantum dot bioconjugates for imaging, labelling and sensing. Nat. Mater. 4, 435-446 (2005).

3. Frecker, T., Bailey, D., Arzeta-Ferrer, X., McBride, J. \& Rosenthal, S. J. Reviewquantum dots and their application in lighting, displays, and biology. ECS J. Solid State Sci. Technol. 5, R3019-R3031 (2016).

4. Won, Y.-H. et al. Highly efficient and stable InP/ZnSe/ZnS quantum dot lightemitting diodes. Nature 575, 634-638 (2019).

5. Tamang, S., Lincheneau, C., Hermans, Y., Jeong, S. \& Reiss, P. Chemistry of InP nanocrystal syntheses. Chem. Mater. 28, 2491-2506 (2016).

6. Xie, R., Battaglia, D. \& Peng, X. Colloidal InP nanocrystals as efficient emitters covering blue to near-infrared. J. Am. Chem. Soc. 129, 15432-15433 (2007).

7. Kim, K-H. et al. Cation-exchange-derived InGaP alloy quantum dots toward blue emissivity. Chem. Mater. 32, 3537-3544 (2020).

8. Kim, Y. et al. Bright and uniform green light emitting InP/ZnSe/ZnS quantum dots for wide color gamut displays. ACS Appl. Nano Mater. 2, 1496-1504 (2019).

9. Jang, E., Kim, Y., Won, Y.-H., Jang, H. \& Choi, S.-M. Environmentally friendly InPbased quantum dots for efficient wide color gamut displays. ACS Energy Lett. 5, 1316-1327 (2020).

10. Gary, D. C., Terban, M. W., Billinge, S. J. L. \& Cossairt, B. M. Two-step nucleation and growth of $\ln P$ quantum dots via magic-sized cluster intermediates. Chem. Mater. 27, 1432-1441 (2015).
11. Kwon, Y. et al. Evolution from unimolecular to colloidal-quantum-dot-like character in chlorine or zinc incorporated InP magic size clusters. Nat. Commun. 11, 3127 (2020).

12. Ning, J. \& Banin, U. Magic size InP and InAs clusters: synthesis, characterization and shell growth. Chem. Commun. (Camb.) 53, 2626-2629 (2017).

13. Xie, L. et al. Characterization of indium phosphide quantum dot growth intermediates using MALDI-TOF mass spectrometry. J. Am. Chem. Soc. 138, 13469-13472 (2016).

14. Chandrasekaran, V. et al. Nearly blinking-free, high-purity single-photon emission by colloidal InP/ZnSe quantum dots. Nano Lett. 17, 6104-6109 (2017).

15. Harris, D. K. \& Bawendi, M. G. Improved precursor chemistry for the synthesis of III-V quantum dots. J. Am. Chem. Soc. 134, 20211-20213 (2012).

16. Franke, D., Harris, D. K., Xie, L., Jensen, K. F. \& Bawendi, M. G. The unexpected influence of precursor conversion rate in the synthesis of III- $V$ quantum dots. Angew. Chem. 54, 14299-14303 (2015).

17. Li, L., Protière, M. \& Reiss, P. Economic synthesis of high quality InP nanocrystals using calcium phosphide as the phosphorus precursor. Chem. Mater. 20, 2621-2623 (2008).

18. Tessier, M. D. et al. Aminophosphines: a double role in the synthesis of colloidal indium phosphide quantum dots. J. Am. Chem. Soc. 138, 5923-5929 (2016).

19. Buffard, A. et al. Mechanistic insight and optimization of inp nanocrystals synthesized with aminophosphines. Chem. Mater. 28, 5925-5934 (2016).

20. An, S., Cho, H., Jeon, H. B. \& Kim, S. W. InPZnS alloy quantum dots with tris (hexylthio)phosphine as a dual anionic precursor. Nanoscale 10, 3014-3019 (2018).

21. Liu, Z. et al. Coreduction colloidal synthesis of III-V nanocrystals: the case of InP. Angew. Chem. 120, 3596-3598 (2008).

22. Carenco, S. et al. White phosphorus and metal nanoparticles: a versatile route to metal phosphide nanoparticles. Chem. Commun. 46, 5578-5580 (2010).

23. Jun, K-W., Khanna, P. K., Hong, K.-B., Baeg, J.-O. \& Suh, Y.-D. Synthesis of InP nanocrystals from indium chloride and sodium phosphide by solution route. Mater. Chem. Phys. 96, 494-497 (2006).

24. Li, Y. et al. Stoichiometry-controlled InP-based quantum dots: synthesis, photoluminescence, and electroluminescence. J. Am. Chem. Soc. 141, 6448-6452 (2019).

25. Ramasamy, P., Ko, K.J., Kang, J.W. \& Lee, J.S. Two-step "seed-mediated" synthetic approach to colloidal indium phosphide quantum dots with highpurity photo- and electroluminescence. Chem. Mater. 30, 3643-3647 (2018).

26. Chen, O. et al. Compact high-quality CdSe-CdS core-shell nanocrystals with narrow emission linewidths and suppressed blinking. Nat. Mater. 12, 445-451 (2013).

27. Thanh, N. T., Maclean, N. \& Mahiddine, S. Mechanisms of nucleation and growth of nanoparticles in solution. Chem. Rev. 114, 7610-7630 (2014).

28. Zhu, T. et al. Two-step nucleation of CdS magic-size nanocluster MSC-311. Chem. Mater. 29, 5727-5735 (2017).

29. Cossairt, B. M. Shining light on indium phosphide quantum dots: understanding the interplay among precursor conversion, nucleation, and growth. Chem. Mater. 28, 7181-7189 (2016).

30. Liu, M. et al. Probing intermediates of the induction period prior to nucleation and growth of semiconductor quantum dots. Nat. Commun. 8 , 15467 (2017).

31. Harrell, S. M., McBride, J. R. \& Rosenthal, S. J. Synthesis of ultrasmall and magicsized CdSe nanocrystals. Chem. Mater. 25, 1199-1210 (2013).

32. Kim, B. H., Hackett, M. J., Park, J. \& Hyeon, T. Synthesis, characterization, and application of ultrasmall nanoparticles. Chem. Mater. 26, 59-71 (2014).

33. Corrigan, J. F., Fuhr, O. \& Fenske, D. Metal chalcogenide clusters on the border between molecules and materials. Adv. Mater. 21, 1867-1871 (2009).

34. Peng, Z. A. \& Peng, X. Nearly monodisperse and shape-controlled CdSe nanocrystals via alternative routes: nucleation and growth. J. Am. Chem. Soc. 124, 3343-3353 (2002).

35. Xie, R., Li, Z. \& Peng, X. Nucleation kinetics vs chemical kinetics in the initial formation of semiconductor nanocrystals. J. Am. Chem. Soc. 131, 15457-15466 (2009).

36. Zhang, L-J., Shen, X-C., Liang, H. \& Yao, J.-T. Multiple families of magic-sized ZnSe quantum dots via noninjection one-pot and hot-injection synthesis. J. Phys. Chem. C. 114, 21921-21927 (2010).

37. Čapek, R. K. et al. Synthesis of extremely small CdSe and bright blue luminescent CdSe/ZnS Nanoparticles by a prefocused hot-injection approach Chem. Mater. 21, 1743-1749 (2009). 
38. Jiang, Z-J. \& Kelley, D. F. Role of magic-sized clusters in the synthesis of CdSe nanorods. ACS Nano 4, 1561-1572 (2010).

39. Beecher, A. N. et al. Atomic structures and gram scale synthesis of three tetrahedral quantum dots. J. Am. Chem. Soc. 136, 10645-10653 (2014).

40. Gao, D. et al. Formation of colloidal alloy semiconductor CdTeSe magic-size clusters at room temperature. Nat. Commun. 10, 1674 (2019).

41. Kudera, S. et al. Sequential growth of magic-size CdSe nanocrystals. Adv. Mater. 19, 548-552 (2007).

42. Chen, H.-S. \& Kumar, R. V. Growth of ultrasmall nanoparticles based on thermodynamic size focusing. J. Nanopart. Res. 14, 1207 (2012).

43. Chen, H. S. \& Kumar, R. V. Discontinuous growth of colloidal CdSe nanocrystals in the magic structure. J. Phys. Chem. C. 113, 31-36 (2009).

44. Chen, H.S. \& Kumar, R. V. Synthesis of ultrasmall quantum dots by redirecting kinetics-based crystal growth to thermodynamics-based crystal dissolution. RSC Adv. 2, 11586-11591 (2012).

45. Gary, D. C. et al. Single-crystal and electronic structure of a $1.3 \mathrm{~nm}$ indium phosphide nanocluster. J. Am. Chem. Soc. 138, 1510-1513 (2016).

46. Friedfeld, M. R. et al. Effects of $\mathrm{Zn}^{2+}$ and $\mathrm{Ga}^{3+}$ doping on the quantum yield of cluster-derived InP quantum dots. J. Chem. Phys. 151, 194702 (2019).

47. Stein, J. L. et al. Cation exchange induced transformation of InP magic-sized clusters. Chem. Mater. 29, 7984-7992 (2017).

48. Yang, J. et al. Route to the smallest doped semiconductor: $\mathrm{Mn}^{2+}$-doped $(\mathrm{CdSe})_{13}$ clusters. J. Am. Chem. Soc. 137, 12776-12779 (2015).

49. Yang, J. et al. $\mathrm{Co}^{2+}$-doping of magic-sized cdse clusters: structural insights via ligand field transitions. Nano Lett. 18, 7350-7357 (2018).

50. Williamson, C. B. et al. Chemically reversible isomerization of inorganic clusters. Science 363, 731-735 (2019).

51. Berrettini, M. G., Braun, G., Hu, J. G. \& Strouse, G. F. NMR analysis of surfaces and interfaces in 2-nm CdSe. J. Am. Chem. Soc. 126, 7063-7070 (2004).

52. Nag, A. et al. Isomerism in supramolecular adducts of atomically precise nanoparticles. J. Am. Chem. Soc. 140, 13590-13593 (2018).

53. Kato, F. \& Kittilstved, K. R. Site-specific doping of $\mathrm{Mn}^{2+}$ in a CdS-based molecular cluster. Chem. Mater. 30, 4720-4727 (2018).

54. Dolai, S. et al. Isolation of bright blue light-emitting CdSe nanocrystals with $6.5 \mathrm{kDa}$ core in gram scale: high photoluminescence efficiency controlled by surface ligand chemistry. Chem. Mater. 26, 1278-1285 (2014).

55. Manju, C. K., Mohanty, J. S., Sarkar, D., Chennu, S. \& Pradeep, T. Towards atomically precise luminescent Ag2S clusters separable by thin layer chromatography. J. Mater. Chem. C. 6, 5754-5759 (2018).

56. Niihori, Y., Uchida, C., Kurashige, W. \& Negishi, Y. High-resolution separation of thiolate-protected gold clusters by reversed-phase high-performance liquid chromatography. Phys. Chem. Chem. Phys. 18, 4251-4265 (2016).

57. Pennycook, T. J., McBride, J. R., Rosenthal, S. J., Pennycook, S. J. \& Pantelides, S. T. Dynamic fluctuations in ultrasmall nanocrystals induce white light emission. Nano Lett. 12, 3038-3042 (2012).

58. Leger, J. D. et al. Carboxylate anchors act as exciton reporters in $1.3 \mathrm{~nm}$ indium phosphide nanoclusters. J. Phys. Chem. Lett. 10, 1833-1839 (2019).

59. Nevers, D. R., Williamson, C. B., Hanrath, T. \& Robinson, R. D. Surface chemistry of cadmium sulfide magic-sized clusters: a window into ligand-nanoparticle interactions. Chem. Commun. (Camb.) 53, 2866-2869 (2017).

60. Cossairt, B. M. \& Owen, J. S. CdSe clusters: at the interface of small molecules and quantum dots. Chem. Mater. 23, 3114-3119 (2011)

61. Nguyen, K. A., Day, P. N. \& Pachter, R. Understanding structural and optical properties of nanoscale CdSe magic-size quantum dots: insight from computational prediction. J. Phys. Chem. C. 114, 16197-16209 (2010).

62. Zhao, Q. \& Kulik, H. J. Electronic structure origins of surface-dependent growth in III-V quantum dots. Chem. Mater. 30, 7154-7165 (2018).

63. Del Ben, M., Havenith, R. W. A., Broer, R. \& Stener, M. Density functional study on the morphology and photoabsorption of CdSe nanoclusters. J. Phys. Chem. C. 115, 16782-16796 (2011).

64. Dmitruk, I. et al. Experimental and computational studies of the structure of CdSe magic-size clusters. J. Phys. Chem. A 124, 3398-3406 (2020).

65. Puzder, A., Williamson, A. J., Gygi, F. \& Galli, G. Self-healing of CdSe nanocrystals: first-principles calculations. Phys. Rev. Lett. 92, 217401 (2004).

66. Beecher, A. N., Dziatko, R. A., Steigerwald, M. L., Owen, J. S. \& Crowther, A. C. Transition from molecular vibrations to phonons in atomically precise cadmium selenide quantum dots. J. Am. Chem. Soc. 138, 16754-16763 (2016).

67. Tan, L. et al. X-ray total scattering study of magic-size clusters and quantum dots of cadmium sulphide. Nanoscale 11, 21900-21908 (2019).
68. Muckel, F. et al. Exciton-driven change of phonon modes causes strong temperature dependent bandgap shift in nanoclusters. Nat. Commun. 11, 4127 (2020).

69. Cumberland, S. L. et al. Inorganic clusters as single-source precursors for preparation of CdSe, ZnSe, and CdSe/ZnS nanomaterials. Chem. Mater. 14, 1576-1584 (2002).

70. Jawaid, A. M., Chattopadhyay, S., Wink, D. J., Page, L. E. \& Snee, P. T. Clusterseeded synthesis of doped CdSe:Cu4 Quantum Dots. ACS Nano 7, 3190-3197 (2013).

71. Ritchhart, A. \& Cossairt, B. M. Templated growth of InP nanocrystals with a polytwistane structure. Angew. Chem. 57, 1908-1912 (2018).

72. Xu, Z. et al. Formation of size-tunable and nearly monodisperse InP nanocrystals: chemical reactions and controlled synthesis. Chem. Mater. 31, 5331-5341 (2019).

73. Tamang, S., Lee, S., Choi, H. \& Jeong, S. Tuning size and size distribution of colloidal inas nanocrystals via continuous supply of prenucleation clusters on nanocrystal seeds. Chem. Mater. 28, 8119-8122 (2016).

74. Xie, R. \& Peng, X. Synthetic scheme for high-quality InAs nanocrystals based on self-focusing and one-pot synthesis of InAs-based core-shell nanocrystals. Angew. Chem. 47, 7677-7680 (2008).

75. Srivastava, V., Dunietz, E., Kamysbayev, V., Anderson, J. S. \& Talapin, D. V. Monodisperse InAs quantum dots from aminoarsine precursors: understanding the role of reducing agent. Chem. Mater. 30, 3623-3627 (2018).

76. Friedfeld, M. R., Johnson, D. A. \& Cossairt, B. M. Conversion of InP clusters to quantum dots. Inorg. Chem. 58, 803-810 (2019).

77. Zhang, J. et al. Evolution of self-assembled ZnTe magic-sized nanoclusters. J. Am. Chem. Soc. 137, 742-749 (2015).

78. Joo, J., Son, J. S., Kwon, S. G., Yu, J. H. \& Hyeon, T. Low-temperature solutionphase synthesis of quantum well structured CdSe nanoribbons. J. Am. Chem. Soc. 128, 5632-5633 (2006).

79. Son, J. S. et al. Large-scale soft colloidal template synthesis of $1.4 \mathrm{~nm}$ thick CdSe nanosheets. Angew. Chem. Int. Ed. 48, 6861-6864 (2009).

80. Kwon, Y., Bang, G., Kim, J., Agnes, A. \& Kim, S. Synthesis of InP branched nanostructures by controlling the intermediate nanoclusters. J. Mater. Chem C. 8, 1118-1124 (2020).

81. Dolai, S., Dutta, P., Muhoberac, B. B., Ivving, C. D. \& Sardar, R. Mechanistic study of the formation of bright white light-emitting ultrasmall CdSe nanocrystals: role of phosphine free selenium precursors. Chem. Mater. 27, 1057-1070 (2015).

82. Rosson, T. E., Claiborne, S. M., McBride, J. R., Stratton, B. S. \& Rosenthal, S. J. Bright white light emission from ultrasmall cadmium selenide nanocrystals. J. Am. Chem. Soc. 134, 8006-8009 (2012).

83. Kucur, E., Ziegler, J. \& Nann, T. Synthesis and spectroscopic characterization of fluorescent blue-emitting ultrastable CdSe clusters. Small 4, 883-887 (2008).

84. Krause, M. M., Mooney, J. \& Kambhampati, P. Chemical and thermodynamic control of the surface of semiconductor nanocrystals for designer white light emitters. ACS Nano 7, 5922-5929 (2013).

85. Bowers, li,M. J. et al. Structure and ultrafast dynamics of white-light-emitting CdSe nanocrystals. J. Am. Chem. Soc. 131, 5730-5731 (2009).

86. Bowers, M. J., McBride, J. R. \& Rosenthal, S. J. White-light emission from magic-sized cadmium selenide nanocrystals. J. Am. Chem. Soc. 127, 15378-15379 (2005).

87. Sadeghi, S., Khabbaz Abkenar, S., Ow-Yang, C. W. \& Nizamoglu, S. Efficient white LEDs using liquid-state magic-sized CdSe quantum dots. Sci. Rep. 9, 10061 (2019).

88. Evans, C. M., Guo, L., Peterson, J. J., Maccagnano-Zacher, S. \& Krauss, T. D. Ultrabright PbSe magic-sized clusters. Nano Lett. 8, 2896-2899 (2008).

89. Lawrence, K. N., Dolai, S., Lin, Y.-H., Dass, A. \& Sardar, R. Enhancing the physicochemical and photophysical properties of small $(<2.0 \mathrm{~nm})$ CdSe nanoclusters for intracellular imaging applications. RSC Adv. 4, 30742-30753 (2014).

90. Mićí, O. I., Ahrenkiel, S. P. \& Nozik, A. J. Synthesis of extremely small InP quantum dots and electronic coupling in their disordered solid films. Appl. Phys. Lett. 78, 4022-4024 (2001).

91. Gary, D. C., Petrone, A., Li, X. \& Cossairt, B. M. Investigating the role of amine in InP nanocrystal synthesis: destabilizing cluster intermediates by Z-type ligand displacement. Chem. Commun. 53, 161-164 (2017).

92. Xie, Y., Teunis, M. B., Pandit, B., Sardar, R. \& Liu, J. Molecule-like CdSe nanoclusters passivated with strongly interacting ligands: energy level alignment and photoinduced ultrafast charge transfer processes. J. Phys. Chem. C. 119, 2813-2821 (2015). 
93. Yang, J. et al. Chemical synthesis, doping, and transformation of magic-sized semiconductor alloy nanoclusters. J. Am. Chem. Soc. 139, 6761-6770 (2017)

94. Antanovich, A., Prudnikau, A., Gurin, V. \& Artemyev, M. Cd/Hg cationic substitution in magic-sized CdSe clusters: optical characterization and theoretical studies. Chem. Phys. 455, 32-40 (2015).

95. Lin, J. et al. Nonlinear variation in the composition and optical band gap of an alloyed cluster-based open-framework metal chalcogenide. Inorg. Chem. 57, 4248-4251 (2018).

96. Lovingood, D. D., Oyler, R. E. \& Strouse, G. F. Composition control and localization of $\mathrm{S}^{2-}$ in $\mathrm{CdSSe}$ quantum dots grown from $\mathrm{Li}_{4}\left[\mathrm{Cd}_{10} \mathrm{Se}_{4}(\mathrm{SPh})_{16}\right]$. J. Am. Chem. Soc. 130, 17004-17011 (2008).

97. $\mathrm{He}, \mathrm{H}$. et al. Ultrasmall $\mathrm{Pb}: \mathrm{Ag}_{2} \mathrm{~S}$ quantum dots with uniform particle size and bright tunable fluorescence in the NIR-II window. Small 14, 1703296 (2018).

98. Eilers, J., Groeneveld, E., de Mello Donegá, C. \& Meijerink, A. Optical properties of Mn-doped ZnTe magic size nanocrystals. J. Phys. Chem. Lett. 3, 1663-1667 (2012).

99. White, S. L., Banerjee, P., Chakraborty, I. \& Jain, P. K. lon exchange transformation of magic-sized clusters. Chem. Mater. 28, 8391-8398 (2016).

100. Fainblat, R., Barrows, C. J. \& Gamelin, D. R. Single magnetic impurities in colloidal quantum dots and magic-size clusters. Chem. Mater. 29, 8023-8036 (2017).

101. Zhang, Q. et al. Exploring $\mathrm{Mn}^{2+}$-location-dependent red emission from (Mn/ $\mathrm{Zn)-Ga-Sn-S} \mathrm{supertetrahedral} \mathrm{nanoclusters} \mathrm{with} \mathrm{relatively} \mathrm{precise} \mathrm{dopant}$ positions. J. Mater. Chem. C. 4, 10435-10444 (2016).

102. Muckel, F. et al. Digital doping in magic-sized CdSe clusters. ACS Nano 10, 7135-7141 (2016).

103. Kim, S. et al. Highly luminescent InP/GaP/ZnS nanocrystals and their application to white light-emitting diodes. J. Am. Chem. Soc. 134, 3804-3809 (2012)

104. Pietra, F. et al. Tuning the lattice parameter of InxZnyP for highly luminescent lattice-matched core/shell quantum dots. ACS Nano 10, 4754-4762 (2016).

105. Zhang, Z. et al. Dual emissive Cu:InP/ZnS/InP/ZnS nanocrystals: single-source "greener" emitters with flexibly tunable emission from visible to near-infrared and their application in white light-emitting diodes. Chem. Mater. 27 1405-1411 (2015).

106. Yang, S., Zhao, P., Zhao, X., Qu, L. \& Lai, X. InP and Sn:InP based quantum dot sensitized solar cells. J. Mater. Chem. A 3, 21922-21929 (2015).

107. Stein, J. L., Mader, E. A. \& Cossairt, B. M. Luminescent InP quantum dots with tunable emission by post-synthetic modification with Lewis acids. J. Phys. Chem. Lett. 7, 1315-1320 (2016)

108. Yu, S. et al. Efficient photocatalytic hydrogen evolution with ligand engineered all-inorganic InP and InP/ZnS colloidal quantum dots. Nat. Commun. 9, 4009 (2018)

109. Kim, K. et al. Halide-amine co-passivated indium phosphide colloidal quantum dots in tetrahedral shape. Angew. Chem. Int. Ed. 55, 3714-3718 (2016)

110. Laufersky, G., Bradley, S., Frecaut, E., Lein, M. \& Nann, T. Unraveling aminophosphine redox mechanisms for glovebox-free InP quantum dot syntheses. Nanoscale 10, 8752-8762 (2018)

111. Bae, W. K. et al. Highly effective surface passivation of PbSe quantum dots through reaction with molecular chlorine. J. Am. Chem. Soc. 134 20160-20168 (2012).

112. Zhang, J. et al. Individual pathways in the formation of magic-size clusters and conventional quantum dots. J. Phys. Chem. Lett. 9, 3660-3666 (2018).

113. Jensen, K. M. et al. Polymorphism in magic-sized Au144(SR)60 clusters. Nat. Commun. 7, 11859 (2016).

114. Okuda, Y. et al. A directly fused subporphyrin dimer with a wavelike structure Angew. Chem. Int. Ed. 55, 9212-9215 (2016).

115. Klimov, V. I. Spectral and dynamical properties of multiexcitons in semiconductor nanocrystals. Annu. Rev. Phys. Chem. 58, 635-673 (2007).

116. Kambhampati, P. Unraveling the structure and dynamics of excitons in semiconductor quantum dots. Acc. Chem. Res. 44, 1-13 (2011).

117. Biadala, L. et al. Band-edge exciton fine structure and recombination dynamics in InP/ZnS colloidal nanocrystals. ACS Nano 10, 3356-3364 (2016).

118. Lim, J. et al. InP@ZnSeS, core@composition gradient shell quantum dots with enhanced stability. Chem. Mater. 23, 4459-4463 (2011).

119. Janke, E. M. et al. Origin of broad emission spectra in $\ln P$ quantum dots: contributions from structural and electronic disorder. J. Am. Chem. Soc. 140 15791-15803 (2018).
120. Stein, J. L. et al. Probing surface defects of InP quantum dots using phosphorus Ka and K $\beta$ X-ray emission spectroscopy. Chem. Mater. 30, 6377-6388 (2018).

121. Friedfeld, M. R., Stein, J. L. \& Cossairt, B. M. Main-group-semiconductor cluster molecules as synthetic intermediates to nanostructures. Inorg. Chem. 56, 8689-8697 (2017).

122. Peng, X. Mechanisms for the shape-control and shape-evolution of colloidal semiconductor nanocrystals. Adv. Mater. 15, 459-463 (2003).

123. Zhang, B. et al. Thermally-induced reversible structural isomerization in colloidal semiconductor CdS magic-size clusters. Nat. Commun. 9, 2499 (2018).

124. Landes, C., Braun, M., Burda, C. \& El-Sayed, M. A. Observation of large changes in the band gap absorption energy of small CdSe nanoparticles induced by the adsorption of a strong hole acceptor. Nano Lett. 1, 667-670 (2001).

125. Wang, R. et al. Single-sized colloidal CdTe nanocrystals with strong bandgap photoluminescence. Chem Commun (Camb) 2009, 962-964 (2009)

126. Wang, $\mathrm{R}$. et al. Magic-sized $\mathrm{Cd}_{3} \mathrm{P}_{2} \|-\mathrm{V}$ nanoparticles exhibiting bandgap photoemission. J. Phys. Chem. C. 113, 17979-17982 (2009).

127. Huang, $M$. et al. Band-emission evolutions from magic-sized clusters to nanosized quantum dots of Cd3As2in the hot-bubbling synthesis. J. Phys. Chem. C. 119, 16390-16395 (2015).

128. Groeneveld, E., van Berkum, S., Meijerink, A. \& de Mello Donega, C. Growth and stability of ZnTe magic-size nanocrystals. Small 7, 1247-1256 (2011).

129. Soloviev, V. N., Eichhöfer, A., Fenske, D. \& Banin, U. Molecular limit of a bulk semiconductor: size dependence of the "band gap" in CdSe cluster molecules. J. Am. Chem. Soc 122, 2673-2674 (2000).

130. Kasuya, A. et al. Ultra-stable nanoparticles of CdSe revealed from mass spectrometry. Nat. Mater. 3, 99-102 (2004).

131. Wang, Y. et al. Isolation of the Magic-Size CdSe Nanoclusters $\left[(\mathrm{CdSe})_{13}(\mathrm{n}-\right.$ octylamine) $\left.{ }_{13}\right]$ and [(CdSe)13(oleylamine)13]. Angew. Chem. Int. Ed. 51, 6154-6157 (2012).

132. Li, M. et al. CdS magic-sized nanocrystals exhibiting bright band gap photoemission via thermodynamically driven formation. ACS Nano 3, 3832-3838 (2009).

133. Chen, X., Samia, A. C. S., Lou, Y. \& Burda, C. Investigation of the crystallization process in $2 \mathrm{~nm}$ CdSe quantum dots. J. Am. Chem. Soc. 127, 4372-4375 (2005).

134. Rockenberger, J. et al. EXAFS studies on the size dependence of structural and dynamic properties of CdS nanoparticles. J. Phys. Chem. B 101 2691-2701 (1997).

135. Soloviev, V. N., Eichhöfer, A., Fenske, D. \& Banin, U. Size-dependent optical spectroscopy of a homologous series of CdSe cluster molecules. J. Am. Chem. Soc. 123, 2354-2364 (2001)

136. Zhu, D. et al. Interpreting the ultraviolet absorption in the spectrum of 415 nm-bandgap CdSe magic-size clusters. J. Phys. Chem. Lett. 9, 2818-2824 (2018).

137. Hsieh, T.-E. et al. Unraveling the structure of magic-size (CdSe) ${ }_{13}$ cluster pairs. Chem. Mater. 30, 5468-5477 (2018).

138. Yu, K. et al. Single-sized CdSe nanocrystals with bandgap photoemission via a noninjection one-pot approach. J. Phys. Chem. C. 113, 3390-3401 (2009).

139. Dukes, A. D., McBride, J. R. \& Rosenthal, S. J. Synthesis of magic-sized CdSe and CdTe nanocrystals with diisooctylphosphinic acid. Chem. Mater. 22, 6402-6408 (2010).

140. Beri, R. K. \& Khanna, P. K. "Green" and controlled synthesis of single family "magic-size" cadmium selenide nanocrystals by the use of cyclo-hexeno1,2,3-selenadiazole an organoselenium compound. CrystEngComm $\mathbf{1 2}$ 2762-2768 (2010).

141. Kilina, S., Ivanov, S. \& Tretiak, S. Effect of surface ligands on optical and electronic spectra of semiconductor nanoclusters. J. Am. Chem. Soc. 131 7717-7726 (2009).

142. Nguyen, K. A., Pachter, R. \& Day, P. N. Computational prediction of structures and optical excitations for nanoscale ultrasmall ZnS and CdSe clusters. J. Chem. Theory Comput. 9, 3581-3596 (2013).

143. Koirala, P. et al. Structural evolution and stabilities of neutral and anionic clusters of lead sulfide: Joint anion photoelectron and computational studies. J. Chem. Phys. 135, 134311 (2011).

144. Park, Y.-S. et al. Size-selective growth and stabilization of small CdSe nanoparticles in aqueous solution. ACS Nano 4, 121-128 (2010). 